\title{
A Novel Integral 5-DOFs Hybrid Magnetic Bearing with One Permanent Magnet Ring Used for Turboexpander
}

\author{
Bangcheng Han, ${ }^{1,2,3}$ Xu Liu, ${ }^{1}$ and Shiqiang Zheng ${ }^{1,2,3}$ \\ ${ }^{1}$ School of Instrument Science and Opto-Electronics Engineering, Beihang University, Beijing 100191, China \\ ${ }^{2}$ Key Laboratory of Fundamental Science for National Defense of Novel Inertial Instrument and Navigation System Technology, \\ Beijing 100191, China \\ ${ }^{3}$ Science and Technology on Inertial Laboratory, Beijing 100191, China
}

Correspondence should be addressed to Bangcheng Han; hanbangcheng@buaa.edu.cn

Received 9 September 2013; Revised 20 January 2014; Accepted 6 February 2014; Published 7 May 2014

Academic Editor: Hector Puebla

Copyright (C) 2014 Bangcheng Han et al. This is an open access article distributed under the Creative Commons Attribution License, which permits unrestricted use, distribution, and reproduction in any medium, provided the original work is properly cited.

We propose a novel combined five-degrees-of-freedom (5-DOFs) hybrid magnetic bearing (HMB) with only one permanent magnet ring (PMR) used for turboexpanders. It has two radial magnetic bearing (RMB) units; each has four poles and one thrust magnetic bearing (TMB) to control 5-DOFs. Based on one PMR, the bias flux of the two radial magnetic bearing units and the one thrust magnetic bearing unit is constructed. As a result, ultra-high-speed, lower power loss, small size, and low cost can be achieved. Furthermore, the equivalent magnetic circuit method and 3D finite element method (FEM) are used to model and analyze the combined 5-DOFs HMB. The force-current, force-position, torque-coil currents, the torque-angle position, and the stiffness models of the combined 5-DOFs HMB are given. Moreover, its coupling problems between the RMB units and the AMB unit are also proposed in this paper. An example is given to clarify the mathematical models and the coupling problems, and the linearized models are proposed for the follow-up controller design.

\section{Introduction}

Magnetic bearing can provide many advantages for turbomachinery such as high-speed, long-life, higher efficiency, lower maintenance, and higher reliability. However, the greatest benefit for turboexpanders is realized by the ability of the magnetic bearings to run in the process gas, which in turn eliminates the lube oil and dry gas seals. As a result, there is no lube oil to contaminate the process. Elimination of the lubrication system also provides significant size and mass savings for off-shore applications. The turboexpanders in S2M, GE, SKF, and so forth have been equipped with the magnetic bearings [1-5]. Jumonville et al. provide useful photographs of actual magnetic bearing hardware, loading plots, and general design guidelines. A neon cryogenic turboexpander with magnetic bearing is developed by Hirai et al. [6]. The experimental equipment of active magnetic bearing on a $150 \mathrm{M}^{3}$ turbo-oxygen gas expander is tested by Xiping et al. [7]. Active magnetic bearing applied on a gas expander is mentioned in the literature $[8,9]$. A magnetically levitated $400 \mathrm{~kW}$ turbogenerator system for natural gas expansion is developed in the literature [10]. Schmied provides excellent information on magnetic bearing control system in a very understandable fashion [11]. The rotor of the turboexpander mentioned above is supported by active magnetic bearings in 5 degrees-of-freedom (DOFs) (Figure 1), which consists of two radial magnetic bearings with bias current, two thrust magnetic bearings with bias current, expander wheel, displacement sensors, shaft, touchdown bearings, and compressor wheel. The radial magnetic bearing and thrust magnetic bearing with bias current have better control ability and high stiffness, whereas they suffer from high power loss due to the bias current. To reduce the power consumption, hotness, and nonlinearity, the radial and axial hybrid magnetic bearings with permanent magnet providing bias flux for suspension and the electromagnet 


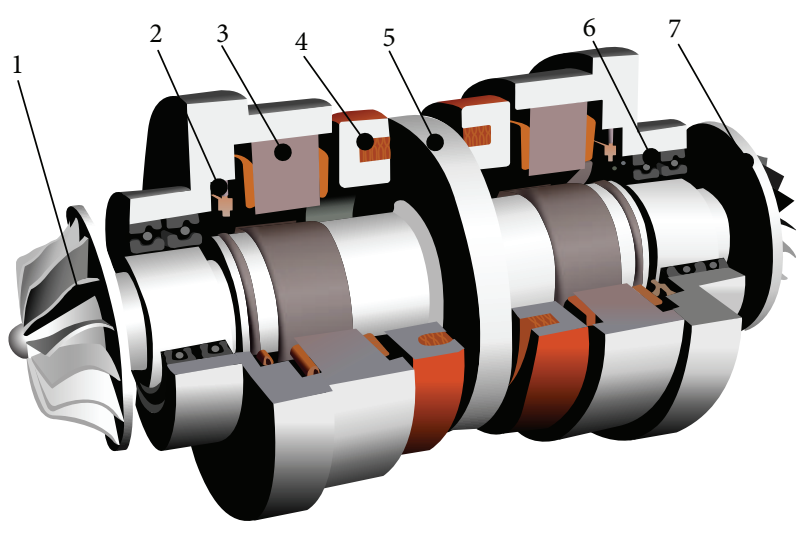

FIGURE 1: The configuration of turboexpander supported by the active magnetic bearings in 5 DOFs. 1: expander wheel; 2: displacement sensor; 3: radial magnetic bearing; 4: thrust magnetic bearing; 5: shaft; 6: touchdown bearing; 7: compressor wheel.

providing necessary control flux are developed [12-15]. Most of the 5-axis hybrid magnetic bearing (HMB) systems are usually composed of two radial hybrid magnetic bearings (RMBs) and one pair of axial hybrid magnetic bearings (AMBs) [16-18]. These magnetic bearing systems are easy to manufacture, but their disadvantages are high power consumption, bulky, complex process, and high cost. The reason is that each RMB has eight poles that lead to more eddy current loss. Moreover, each of RMBs and AMBs has one permanent magnet ring (PMR) that is used to provide bias flux. As many as four PMRs are required in this 5-DOFs HMB system.

In order to reduce the power consumption, cost, and size, it is important to reduce the number of the units by means of combining the RMB and AMB. Integrated axial magnetic bearing and radial magnetic bearing with the conical rotor are designed and analyzed $[19,20]$ and one downside of the integrated bearings is a strong coupling problem between the radial and axial degrees-of-freedom. In addition, the combined radial-axial hybrid magnetic bearings (CRAMBs) are proposed [21-28]. The CRAMBs mentioned above have been applied in ultra-high-speed machinery, but it may not suit the turboexpanders.

In this paper, a novel combined 5-DOFs HMB with only one PMR is proposed to be used for turboexpanders in this paper. The combined 5-DOFs HMB consists of two RMB units each with four poles and one AMB unit. The bias flux of the two RMB units and AMB unit is supplied by only one PMR. The low power consumption, compact structure, and small size can be gained due to the combined 5-DOFs HMB, the permanent magnetic bias $\mathrm{HMB}$, and the reduction in the number of the poles of the RMB units. The low cost is gained due to the one PMR used. Moreover, ultra-high-speed is also gained due to the small outside diameter of the rotor thrust disk. The equivalent magnetic circuit method and 3D finite element method (FEM) are used to model and analyze the combined 5-DOFs HMB. The force-current, force-position, torque-current, the torque-angular position, and the stiffness

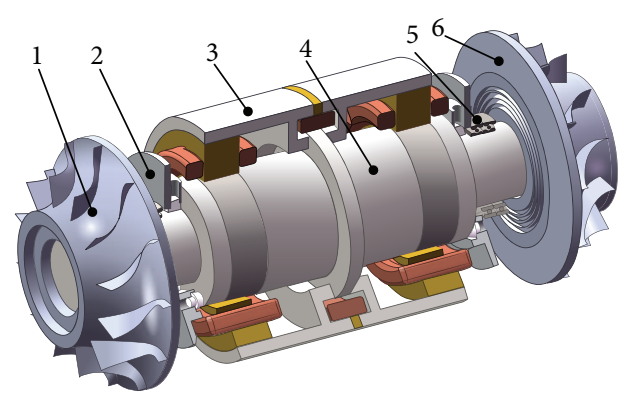

FIGURE 2: The configuration of turboexpander supported by the combined 5-DOFs HMB. 1: expander wheel; 2: displacement sensor; 3: the combined 5-DOFs HMB; 4: shaft; 5: touchdown bearing; 6: compressor wheel.

models of the integral 5-DOFs HMB are given. Moreover, its coupling problems between the RMB units and the AMB unit are also proposed. An example is given to clarify the mathematical models and the coupling problems, and the linearized models are proposed for the follow-up controller design.

This paper is organized as follows. First, in Section 2, the configuration of combined 5-DOFs HMB is given. Then, the equivalent magnetic circuit method is used to model and analyze the combined 5-DOFs HMB in Section 3. Section 4 is devoted to developing simulation and clarifying the linearized models. Finally, Section 5 concludes this paper. We focus on the configuration, modeling, and analysis of the combined 5-DOFs HMB; a future paper will compare with experimental results.

\section{Analyzed Configuration}

A simplified configuration of turboexpander supported by the combined 5-DOFs HMB is shown in Figure 2. The assembly consists of one 5-DOFs HMB, expander wheel, displacement sensor, shaft, touchdown bearing, and compressor wheel. And the rigid body motion on the rotorbearing system is considered in this paper. The configuration and magnetic circuit of the combined 5-DOFs HMB are shown in Figure 3. The force and torque produced by the RMB units and $A M B$ unit are shown in Figure 4. It consists of two RMB units (RMB units $A$ and $B$ ), one pair of $A M B$ unit, and one axially magnetized PMR. The bias fluxes produced by the permanent magnets are shown by the solid arrow lines and the control flux is generated by $y$-axis (blue color) and $z$-axis coil currents (red color), which are shown by the dotted arrow lines in Figure 3. Each stator of the RMB unit consists of two soft magnetic pieces each of which has four equally spaced magnetic poles and four coils distributed at intervals of 90 degrees around the stator iron. The control flux produced by the radial and axial coil currents will allow adding or subtracting flux to the radial air gaps and axial air gaps, and then restoring force and torque will be produced to stabilize the rotor in 5-DOFs except the spin axis in relation 


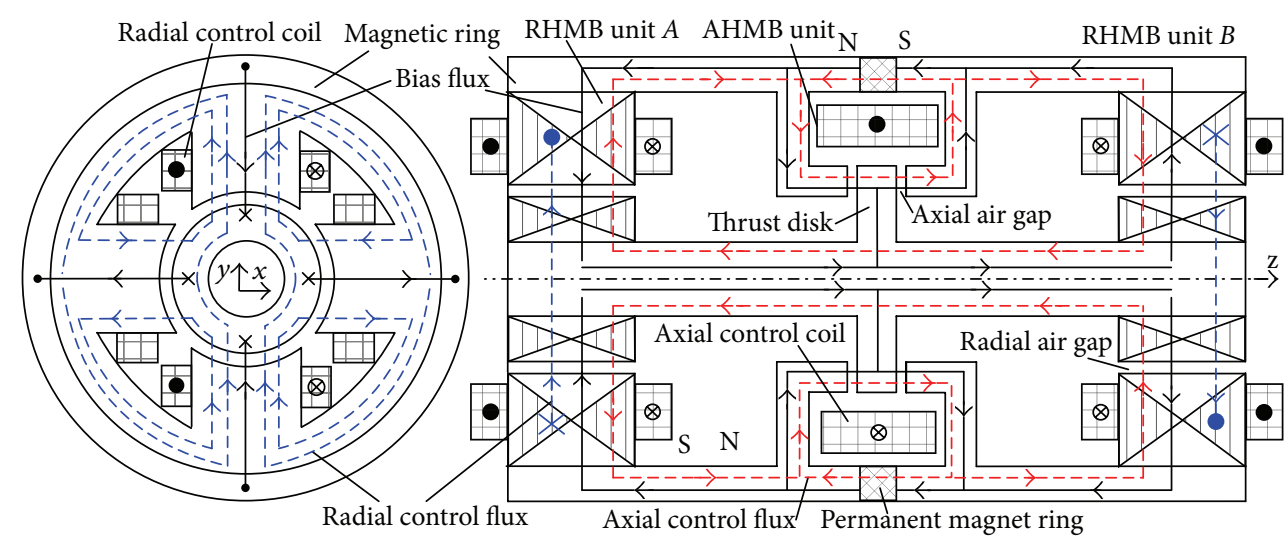

(a)

(b)

Figure 3: The configuration and magnetic circuit of the combined 5-DOFs HMB. (a) The section view of the RMB unit $A$. (b) The $y-z$ section view of the combined 5-DOFs HMB.

to the rotor position. The bias flux of the RMB units and AMB unit is produced by only one PMR. The RMB units $A$ and $B$ stabilize rotor on the two axes ( $x$-axis and $y$-axis) orthogonal to the spin axis ( $z$-axis) and exert restoring torque on the rotor around $x$-axis or (and) $y$-axis when subjected to tilting disturbed torque. The AMB unit exerts restoring force on the rotor at $z$-axis. In a word, the low power consumption, compact structure, and small size can be gained due to the combined 5-DOFs HMB, the permanent magnetic bias HMB, and the reduction in the number of the poles of the RMB unit. The low cost is gained due to only one PMR being used. Moreover, ultra-high-speed is also gained due to the small outside diameter of the rotor thrust disk.

\section{Modeling and Analysis of the Combined 5-DOFs HMB}

3.1. The Equivalent Magnetic Circuits of the Combined 5-DOFs $H M B$. According to construction of the combined 5-DOFs $\mathrm{HMB}$, the magnetic resistance of the iron path and flux leakage is neglected, the permeability of the iron is assumed to be infinite, and their equivalent bias and control magnetic circuits can be gained and are shown in Figure 5.

\subsection{The Model of Combined 5-DOFs HMB for Translation in $x$ -} Axis (or $y$-Axis) and $z$-Axis Direction. Figure 3 describes the operating principle of this combined 5-DOFs HMB in $y$-axis and $z$-axis. The rotor will depart from its center position when subject disturbance force in negative $y$-axis direction. The control flux on the upper side of the RMB units $A$ and $B$ will add to the bias flux in upper radial air gap and subtract from the bias flux in the lower radial air gap. And the control flux of the AMB unit should also be calculated. A net restoring force on the rotor will be produced in positive $y$-axis direction.

According to the structure of the combined 5-DOFs HMB (Figure 4) and its equivalent magnetic circuits (Figure 5), the net force of the RMB unit $\mathrm{A}$ in the $y$-axis direction

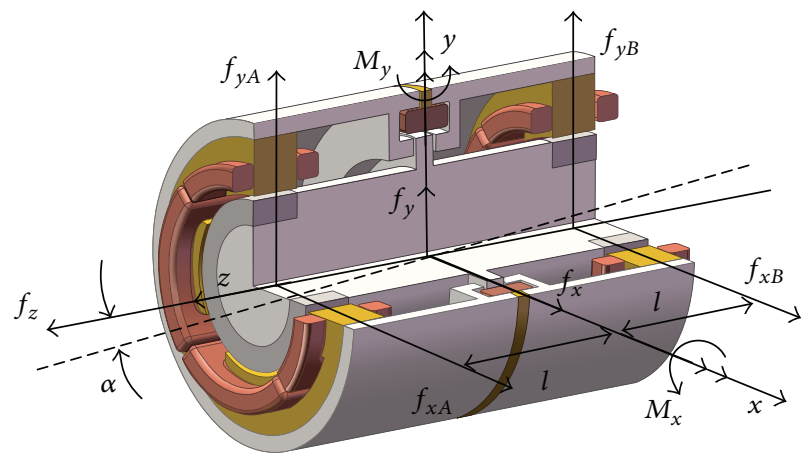

FIGURE 4: The force and moment produced by the combined 5DOFs HMB.

$\left(N_{r} i_{A x}=0\right.$, where $N_{r}$ is the number of winding turns and $i_{A x}$ is the control current of $x$-axis coil currents), $f_{y}$, is

$$
f_{A y}=\frac{\left(\phi_{y+}^{A p}+\phi_{y+}^{A i}-\phi_{y+}^{A z}\right)^{2}}{2 \mu_{0} A_{r}}-\frac{\left(\phi_{y-}^{A p}-\phi_{y-}^{A i}-\phi_{y-}^{A z}\right)^{2}}{2 \mu_{0} A_{r}},
$$

where the constant $\mu_{0}$ is the permeability of free space and is equal to $4 \pi \times 10^{-7} \mathrm{H} / \mathrm{m} ; A_{r}$ is the area of the pole face of RMB unit; $\phi_{y+}^{A p}$ and $\phi_{y-}^{A p}$ are the bias flux in radial air gaps of the RMB unit $A ; \phi_{y+}^{A i}$ and $\phi_{y-}^{A i}$ are the control flux in the radial air gaps of the RMB unit $A$ produced by the $y$-axis coil currents; $\phi_{y+}^{A z}$ and $\phi_{y-}^{A z}$ are the control flux in the radial air gaps of the RMB unit $A$ produced by the $z$-axis coil current of the AMB unit. follows:

The bias flux in Figure 5 can be calculated, respectively, as

$$
\begin{aligned}
& \phi_{y+}^{A p}=\frac{F_{m}}{R_{\text {sum }}} \frac{R_{A} R_{z 1}}{R_{A}+R_{z 1}} \frac{1}{R_{y+}^{A}}, \\
& \phi_{y-}^{A p}=\frac{F_{m}}{R_{\text {sum }}} \frac{R_{A} R_{z 1}}{R_{A}+R_{z 1}} \frac{1}{R_{y-}^{A}},
\end{aligned}
$$




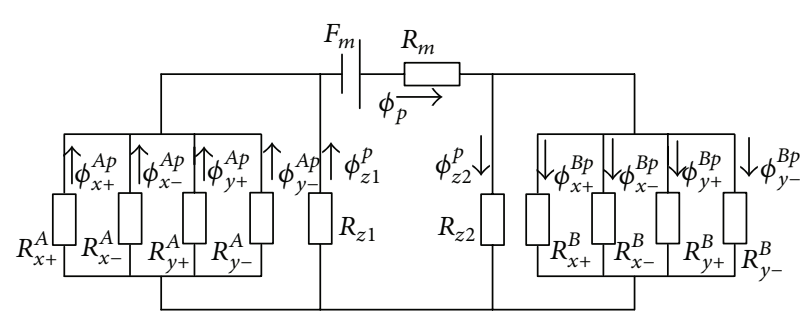

(a)

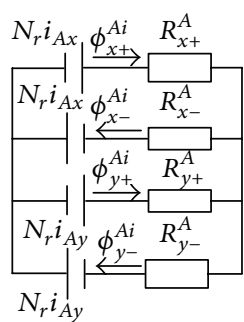

(b)

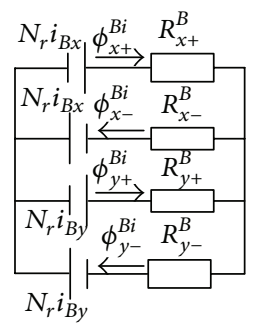

(c)

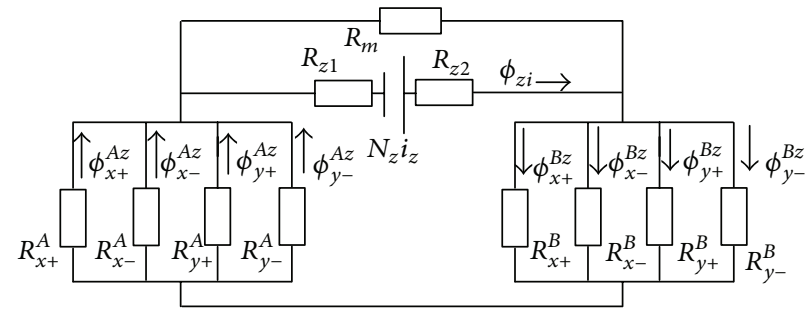

(d)

FIGURE 5: Equivalent bias circuit and control circuits of the combined 5-DOFs HMB. (a) The equivalent bias circuit. (b) The equivalent control circuit of RMB unit $A$. (c) The equivalent control circuit of RMB unit $B$. (d) The equivalent control circuit of AMB unit.

$$
\begin{aligned}
\phi_{z 1}^{p} & =\frac{F_{m}}{R_{\text {sum }}} \frac{R_{A} R_{z 1}}{R_{A}+R_{z 1}} \frac{1}{R_{z 1}}, \\
\phi_{z 2}^{p} & =\frac{F_{m}}{R_{\text {sum }}} \frac{R_{B} R_{z 2}}{R_{B}+R_{z 2}} \frac{1}{R_{z 2}},
\end{aligned}
$$

where

$$
\begin{aligned}
R_{\text {sum }} & =R_{m}+\frac{R_{A} R_{z 1}}{R_{A}+R_{z 1}}+\frac{R_{B} R_{z 2}}{R_{B}+R_{z 2}}, \\
R_{A} & =\frac{1}{\left(1 / R_{x+}^{A}\right)+\left(1 / R_{x-}^{A}\right)+\left(1 / R_{y+}^{A}\right)+\left(1 / R_{y-}^{A}\right)}, \\
R_{B} & =\frac{1}{\left(1 / R_{x+}^{B}\right)+\left(1 / R_{x-}^{B}\right)+\left(1 / R_{y+}^{B}\right)+\left(1 / R_{y-}^{B}\right)} .
\end{aligned}
$$

As shown in Figures 3 and $5, R_{\text {sum }}$ is the total reluctance of the bias circuit; $R_{x+}^{A}$ and $R_{x-}^{A}$ are the reluctance of the radial air gaps in $x$-axis for the RMB unit $A ; R_{y+}^{A}$ and $R_{y-}^{A}$ are the reluctance of the radial air gaps in $y$-axis for the RMB unit $A ; R_{x+}^{B}$ and $R_{x-}^{B}$ are the reluctance of the radial air gaps in $x$ axis for the RMB unit $B ; R_{y+}^{B}$ and $R_{y-}^{B}$ are the reluctance of the radial air gaps in $y$-axis for the RMB unit $B ; R_{z 1}$ and $R_{z 2}$ are the reluctance of the axial air gaps in $z$-axis for the AMB unit. $F_{m}=H_{c} h_{m}$, where $H_{c}$ is the coercive force of the permanent magnet; $h_{m}$ is PMR thickness; $R_{m}$ is the magnetic resistance of PMR and it can be given as

$$
R_{m}=\frac{h_{m}}{\mu_{0} \mu_{r} A_{m}},
$$

where $\mu_{r}$ is the relative permeability of permanent magnet and $A_{m}$ is the middle-section area of the PMR.
The reluctances of radial air gap are written by

$$
\begin{array}{ll}
R_{x+}^{A}=\frac{s_{r 0}+x}{\mu_{0} A_{r}}, & R_{x-}^{A}=\frac{s_{r 0}-x}{\mu_{0} A_{r}}, \\
R_{x+}^{B}=\frac{s_{r 0}+x}{\mu_{0} A_{r}}, & R_{x-}^{B}=\frac{s_{r 0}-x}{\mu_{0} A_{r}}, \\
R_{y+}^{A}=\frac{s_{r 0}+y}{\mu_{0} A_{r}}, & R_{y-}^{A}=\frac{s_{r 0}-y}{\mu_{0} A_{r}}, \\
R_{y+}^{B}=\frac{s_{r 0}+y}{\mu_{0} A_{r}}, & R_{y-}^{B}=\frac{s_{r 0}-y}{\mu_{0} A_{r}},
\end{array}
$$

where $s_{r 0}$ is the nominal air gap of the RMB unit; $y$ is the rotor position as deviation from the operating point in $y$-axis direction; $x$ is the deviation from the operating point in $x$-axis direction.

The reluctances of axial air gap are written by

$$
R_{z 1}=\frac{s_{z 0}+z}{\mu_{0} A_{z}}, \quad R_{z 2}=\frac{s_{z 0}-z}{\mu_{0} A_{z}},
$$

where $s_{z 0}$ is the nominal air gap of the AMB unit; $z$ is the rotor position as deviation from the operating point in $z$-axis direction; $A_{z}$ is the area of the pole face for the AMB unit.

The control flux in the radial air gaps of $y$-axis, $\phi_{y+}^{A i}$ and $\phi_{y-}^{A i}$, generated by $y$-axis coil currents of RMB unit $A\left(N_{r} i_{A x}=0\right.$, where $N_{r}$ is the number of winding turns and 
$i_{A x}$ is the control current of $x$-axis coil currents) can be calculated as

$$
\begin{aligned}
\phi_{y+}^{A i}= & \frac{N_{r} i_{A y}}{R_{y+}^{A}+R_{x-}^{A} / / R_{x+}^{A} / / R_{y-}^{A}} \\
& +\frac{N_{r} i_{A y}}{R_{y-}^{A}+R_{x-}^{A} / / R_{x+}^{A} / / R_{y+}^{A}} \frac{R_{x-}^{A} / / R_{x+}^{A} / / R_{y+}^{A}}{R_{y+}^{A}}, \\
\phi_{y-}^{A i}= & \frac{N_{r} i_{A y}}{R_{y-}^{A}+R_{x-}^{A} / / R_{x+}^{A} / / R_{y+}^{A}} \\
& +\frac{N_{r} i_{A y}}{R_{y+}^{A}+R_{x-}^{A} / / R_{x+}^{A} / / R_{y-}^{A}} \frac{R_{x-}^{A} / / R_{x+}^{A} / / R_{y-}^{A}}{R_{y-}^{A}},
\end{aligned}
$$

where $i_{A y}$ is the control current of $y$-axis coil currents for the same displacement of RMB unit $A$.

The control flux in the radial air gaps, $\phi_{y+}^{A z}$ and $\phi_{y-}^{A z}$, which are produced by the $z$-axis coil currents of the AMB unit can be calculated as

$$
\begin{aligned}
& \phi_{y+}^{A z}=\phi_{z i}\left[R_{m} / /\left(R_{A}+R_{B}\right)\right] \frac{R_{A}}{R_{A}+R_{B}} \frac{1}{R_{y+}^{A}}, \\
& \phi_{y-}^{A z}=\phi_{z i}\left[R_{m} / /\left(R_{A}+R_{B}\right)\right] \frac{R_{A}}{R_{A}+R_{B}} \frac{1}{R_{y-}^{A}},
\end{aligned}
$$

where the control flux in the axial air gap is calculated by

$$
\phi_{z i}=\frac{N_{z} i_{z}}{R_{z 1}+R_{z 2}+R_{m} / /\left(R_{A}+R_{B}\right)},
$$

where $N_{z}$ is the number of winding turns of the AMB and $i_{z}$ is the control current of $z$-axis coil current. And $\phi_{z i 1}=\phi_{z i 2}$.

Substituting (2), (7), and (8) into the right side of (1) results in the net force on $y$-axis direction. Using the Taylor series expansion for small values of $y$ and $i_{y}$, we can get the following attractive force with linear terms in $y$-axis direction:

$$
\left.f_{A y}\left(y, i_{y}\right) \cong f_{A y}\right|_{\substack{x=y=z=0 \\ i_{A y}=i_{z}=0}}+k_{r i} i_{y}+k_{r s} y
$$

Since the net force is zero at the center position in radial and axial directions, (10) can be written by

$$
f_{A y}\left(y, i_{A y}\right)=k_{r i} i_{y}+k_{r s} y
$$

where $k_{r i}$ is the current stiffness of the RMB unit and $k_{r s}$ is the negative position stiffness of the RMB unit caused by the increasing attractive force as the air gap is reduced. This must be overcome for stable suspension since the homogeneous solution to magnetic bearing force $(\mathrm{MBF})$ is in the form of hyperbolic functions indicating that $y$ grows with time. They can be calculated as follows:

$$
\begin{aligned}
k_{r i}= & \left.\frac{\partial f_{A y}}{\partial i_{A y}}\right|_{\substack{i_{A y}=i_{z}=0 \\
x=y=0}} \\
= & 2 \mu_{0} A_{r} N_{r} H_{c} h_{m} \\
& \times\left(s_{r 0}^{2}\left(\frac{4 A_{r}}{s_{r 0}}+\frac{A_{z}}{s_{z 0}}\right)\right. \\
& \left.\times\left(\frac{h_{m}}{\mu_{r} A_{m}}+\frac{2 s_{r 0} s_{z 0}}{4 A_{r} s_{z 0}+A_{z} s_{r 0}}\right)\right)^{-1}, \\
k_{r s}= & \left.\frac{\partial f_{A y}}{\partial y}\right|_{\substack{i_{A y}=i_{z}=0 \\
x=y=z=0}}-2 \mu_{0} A_{r}\left(H_{c} h_{m}\right)^{2} \\
& \times\left(s_{r 0}^{3}\left(\frac{4 A_{r}}{s_{r 0}}+\frac{A_{z}}{s_{z 0}}\right)^{2}\right. \\
& \left.\times\left(\frac{h_{m}}{\mu_{r} A_{m}}+\frac{2 s_{r 0} s_{z 0}}{4 A_{r} s_{z 0}+A_{z} s_{r 0}}\right)^{2}\right)^{-1} .
\end{aligned}
$$

According to the structure of the combined 5-DOFs HMB (Figure 4) and its equivalent magnetic circuits (Figure 5), the net force of the AMB unit in the $z$-axis direction is

$$
f_{z}=\frac{\left(\phi_{z 1}^{p}+\phi_{z i}\right)^{2}}{2 \mu_{0} A_{z}}-\frac{\left(\phi_{z 2}^{p}-\phi_{z i}\right)^{2}}{2 \mu_{0} A_{z}},
$$

where $\phi_{z 1}^{p}$ and $\phi_{z 2}^{p}$ are the bias flux in axial air gaps of the AMB unit and $\phi_{z i}$ is the control flux in the axial air gaps of the AMB unit by the $z$-axis coil current.

Substituting (2) and (9) for the right side of (13) results in the net force on $z$-axis direction. Using the Taylor series expansion for small values of $z$ and $i_{z}$, we can get the following attractive force with linear terms in $z$-axis direction:

$$
\left.f_{z}\left(z, i_{z}\right) \cong f_{z}\right|_{\substack{x=y=z=0 \\ i_{A y}=i_{z}=0}}+k_{z i} i_{z}+k_{z s} z
$$
by

Since the net force is zero at the center, (14) can be written

$$
f_{z}\left(z, i_{z}\right)=k_{z i} i_{z}+k_{z s} z
$$

where $k_{z i}$ is the current stiffness of the AMB unit and $k_{z s}$ is the negative position stiffness of the AMB unit caused by the increasing attractive force as the air gap is reduced. This must be overcome for stable suspension since the homogeneous solution to $\mathrm{MBF}$ is in the form of hyperbolic functions 


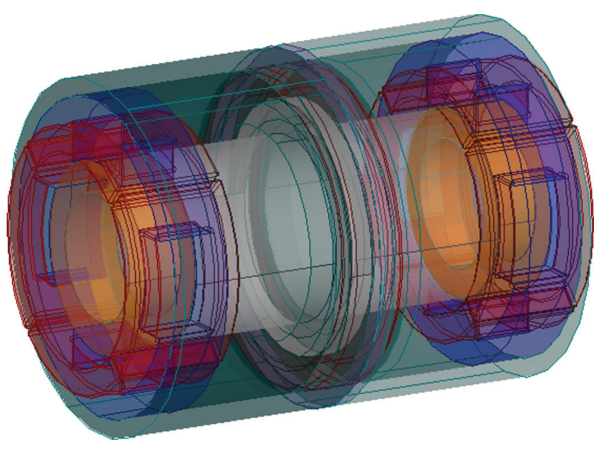

(a)

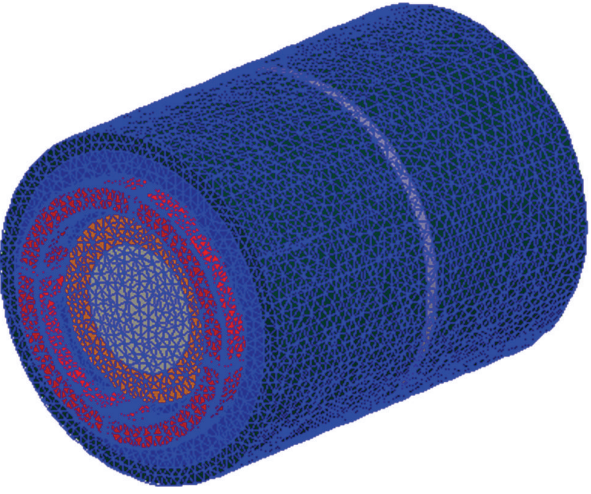

(b)

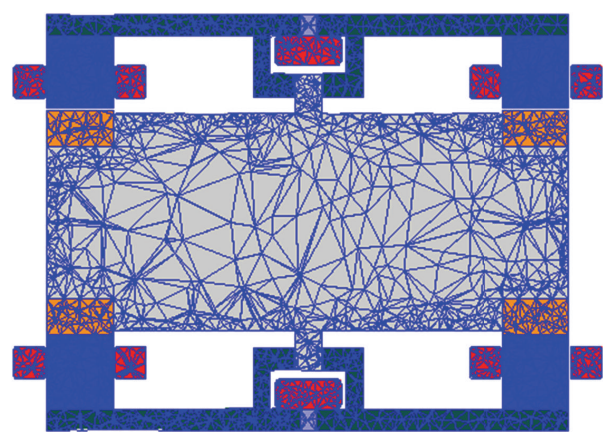

(c)

Figure 6: The 3D finite element model and mesh of the 5-DOFs HMB except the air. (a) The 3D finite element model. (b) The 3D finite element mesh. (c) Section view of the 3D finite element mesh.

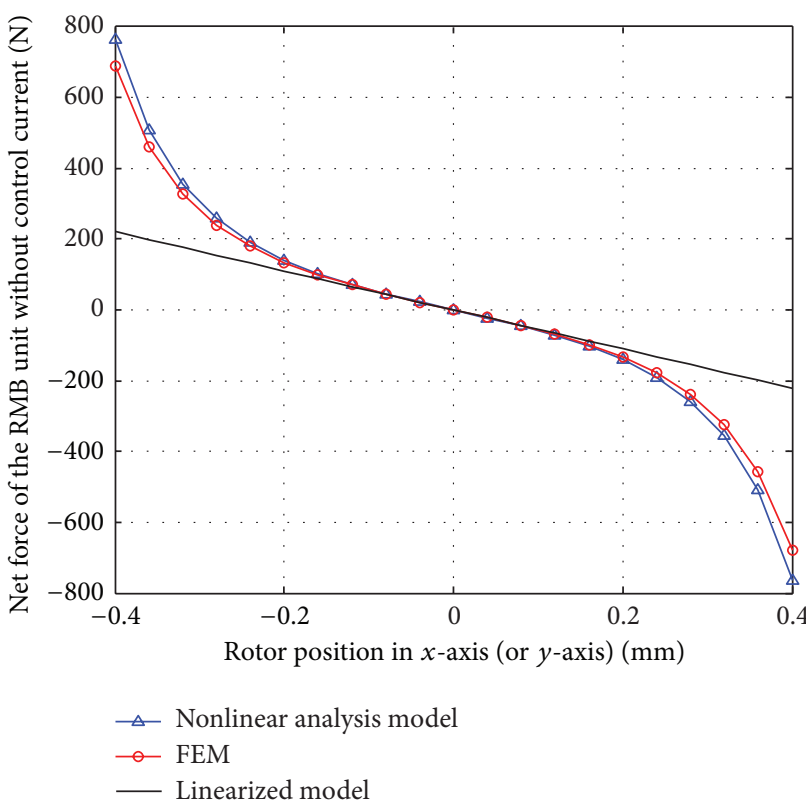

(a)

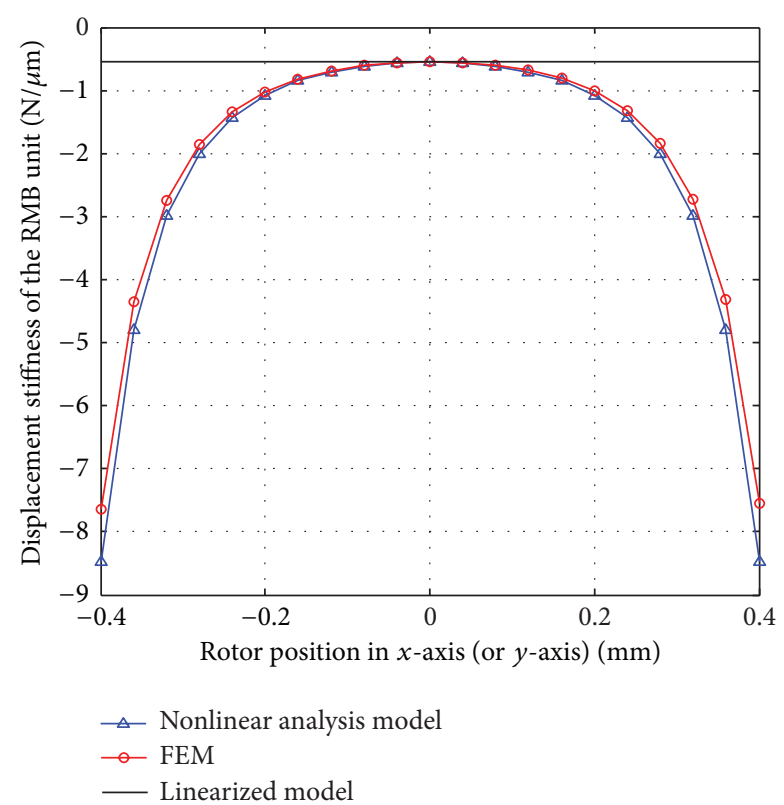

(b)

Figure 7: Curves of the force-rotor position and displacement stiffness in $x$-axis (or $y$-axis) of the RMB unit (using linearized method, nonlinear method, and FEM). (a) Curves of the force-rotor position of the RMB unit. (b) Curves of the displacement stiffness of the RMB unit. 


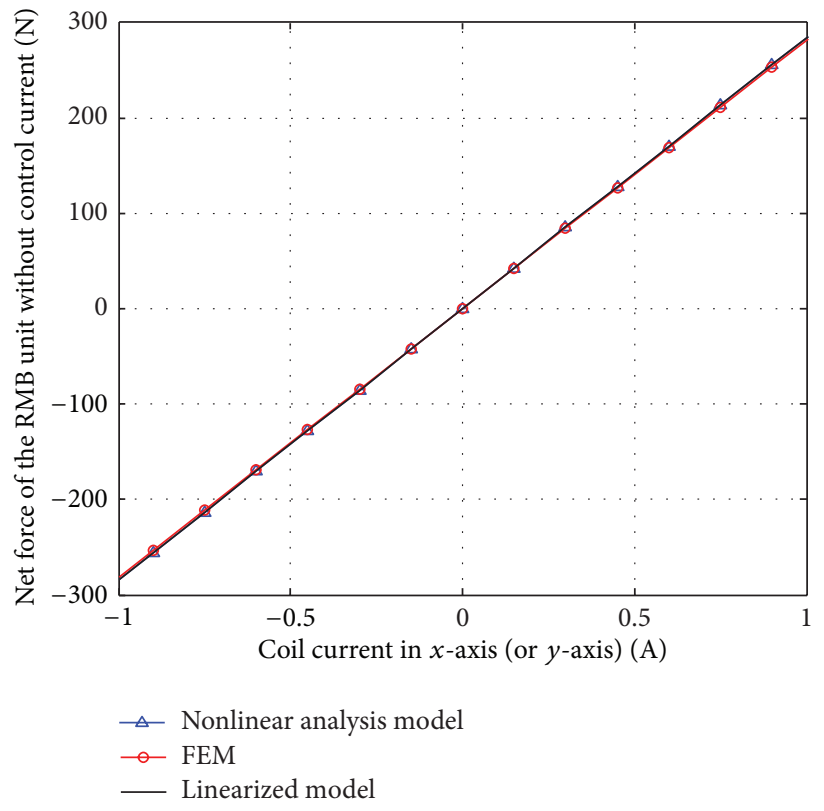

FIGURE 8: Curves of the force-coil current in $x$-axis (or $y$-axis) of the RMB unit (using linearized method, nonlinear method, and FEM).

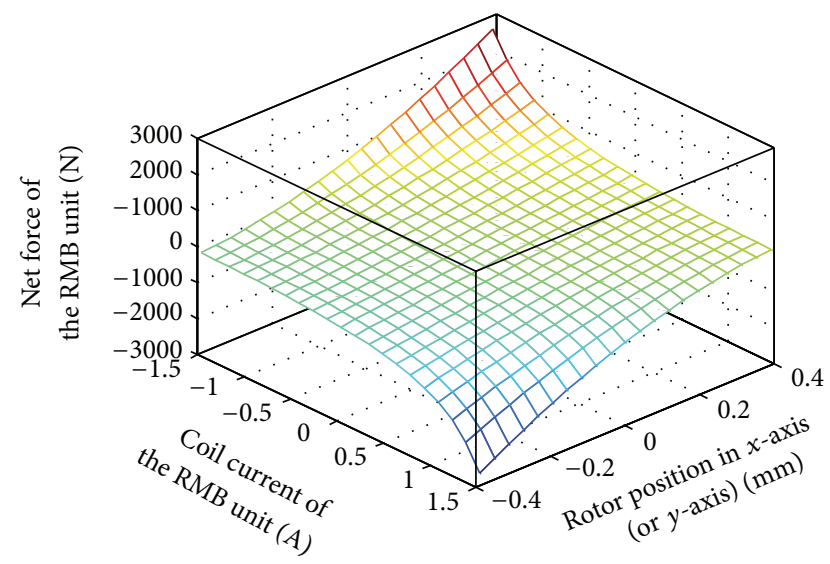

Figure 9: Curves of the force-coil current and rotor position in $x$ axis (or $y$-axis) of the RMB unit (using nonlinear method).

indicating that $z$ grows with time. They can be calculated as follows:

$$
\begin{aligned}
k_{z i}= & \left.\frac{\partial f_{z}}{\partial i_{z}}\right|_{\substack{i_{A y}=i_{B y}=i_{z}=0 \\
x=y=z=0}} \\
= & 2 \mu_{0} N_{z} H_{c} h_{m} \\
& \times\left(\left(\frac{4 A_{r} s_{z 0}}{s_{r 0}}+A_{z}\right)\right. \\
& \times\left(\frac{h_{m}}{\mu_{r} A_{m}}+\frac{2 s_{r 0} s_{z 0}}{4 A_{r} s_{z 0}+A_{z} s_{r 0}}\right)
\end{aligned}
$$

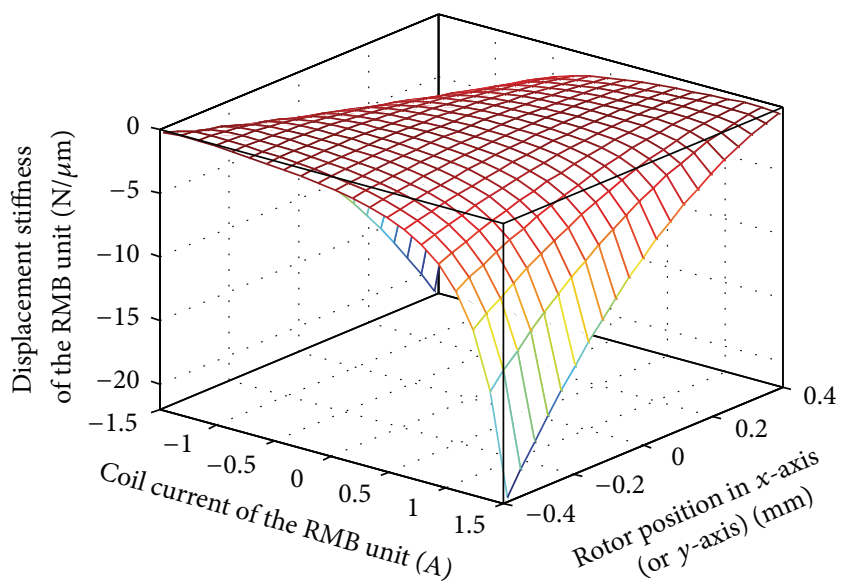

FIgURE 10: Curves of the displacement stiffness-rotor position-coil current and rotor position in $x$-axis (or $y$-axis) of the RMB unit (using nonlinear method).

$$
\begin{gathered}
\left.k_{z s}=\left.\frac{\partial f_{z}}{\partial z}\right|_{\substack{i_{A y}=i_{B y}=i_{z}=0 \\
x=y=z=0}} \times\left(\frac{h_{m} s_{r 0}}{\mu_{r} A_{m} s_{r 0}+2 A_{r} h_{m}}+\frac{2 s_{z 0}}{A_{z}}\right)\right)^{-1}, \\
=\frac{2 \mu_{0} A_{z}\left(H_{c} h_{m}\right)^{2}}{\left(\left(h_{m} / \mu_{r} A_{m}\right)+\left(2 s_{r 0} s_{z 0} /\left(4 A_{r} s_{z 0}+A_{z} s_{r 0}\right)\right)\right)^{2}} \\
\times \frac{A_{z} s_{r 0}^{3}-s_{r 0}^{2}\left(4 A_{r} s_{z 0}+A_{z} s_{r 0}\right)}{s_{z 0}\left(4 A_{r} s_{z 0}+A_{z} s_{r 0}\right)^{3}} .
\end{gathered}
$$




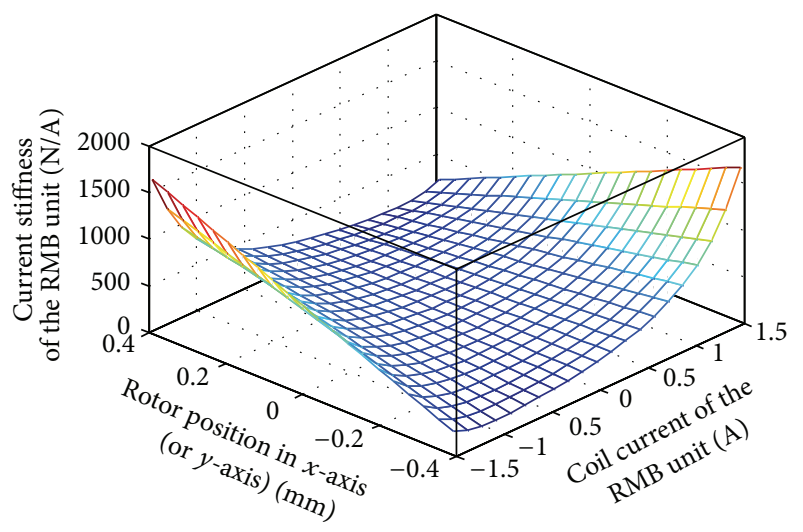

FIgURE 11: Curves of the current stiffness-coil current and rotor position in $x$-axis (or $y$-axis) of the RMB unit (using nonlinear method).

3.3. The Model of Combined 5-DOFs HMB for Tilting. The RMB units $A$ and $B$ of the combined 5-DOFs HMB also control 2-DOFs around $x$-axis and $y$-axis which are orthogonal to the spin axis ( $z$-axis) when the rotor is subject to disturbance torque around $x$-axis and $y$-axis. The RMB units $A$ and $B$ create tilting stiffness to restrain the rotor when subjected to the disturbed torques as shown in Figure 4. According to the structure of the combined 5-DOFs HMB (Figure 3 ) and its equivalent magnetic circuits (Figure 5), the torque, $M_{x}$, acting on the rotor can be given by (17) $\left(N_{z} i_{z}=0\right.$ and $N_{r} i_{A x}=N_{r} i_{B x}=0$ ). Consider

$$
\begin{aligned}
M_{x}=l \cos \alpha & {\left[\frac{\left(\phi_{y+}^{A p}+\phi_{y+}^{A i}\right)^{2}-\left(\phi_{y-}^{A p}-\phi_{y-}^{A i}\right)^{2}}{2 \mu_{0} A_{r}}\right.} \\
& \left.+\frac{\left(\phi_{y-}^{B p}+\phi_{y-}^{B i}\right)^{2}-\left(\phi_{y+}^{B p}-\phi_{y+}^{B i}\right)^{2}}{2 \mu_{0} A_{r}}\right],
\end{aligned}
$$

where $l$ is the distance from center of the magnetic pole of the RMB units $A$ and $B$ to the center of rotor as shown in Figure 4 . The $l * \cos \alpha \cong l$ for small value of the tilting angle, $\alpha$. Equation (17) can be simplified as

$$
M_{x} \cong l\left[\frac{\left(\phi_{y+}^{A p}+\phi_{y+}^{A i}\right)^{2}-\left(\phi_{y-}^{A p}-\phi_{y-}^{A i}\right)^{2}}{\mu_{0} A_{r}}\right] \text {. }
$$

The change in radial air gap is $l * \tan \alpha \cong l * \alpha$ for small value of the tilting angle, $\alpha$. The radial air gap resistance can be written as

$$
\begin{aligned}
R_{x+}^{A}=\frac{s_{r 0}}{\mu_{0} A_{r}}, & R_{x-}^{A}=\frac{s_{r 0}}{\mu_{0} A_{r}} \\
R_{x+}^{B}=\frac{s_{r 0}}{\mu_{0} A_{r}}, & R_{x-}^{B}=\frac{s_{r 0}}{\mu_{0} A_{r}}, \\
R_{y+}^{A}=R_{y-}^{B} \cong \frac{s_{r 0}+l \alpha}{\mu_{0} A_{r}}, & R_{y-}^{A}=R_{y+}^{B} \cong \frac{s_{r 0}-l \alpha}{\mu_{0} A_{r}} .
\end{aligned}
$$

Using the Taylor series expansion for small values of $\alpha$ and $i_{A y}$ we can get the following attractive torque with linear terms:

$$
M_{x}\left(i_{A y}, \alpha\right) \cong k_{\alpha i} i_{A y}+k_{\alpha s} \alpha
$$

where $k_{\alpha i}$ is the tilting current stiffness and $k_{\alpha s}$ is the negative tilting displacement stiffness. They can be calculated as follows:

$$
\begin{aligned}
k_{\alpha i}= & \left.\frac{\partial M_{x}}{\partial i_{A y}}\right|_{\substack{i_{A y}=0 \\
\alpha=0}} \\
= & 4 \mu_{0} A_{r} N_{r} H_{c} h_{m} l \\
& \times\left(\frac{4 A_{r} s_{z 0} s_{r 0}+A_{z} s_{r 0}^{2}}{s_{z 0}}\right. \\
k_{\alpha s}= & \left.\left.\left.\frac{\partial M_{x}}{\partial \alpha}\right|_{i_{A y}=0} ^{\alpha=0}{ }^{\mu_{r} A_{m}}+\frac{2 s_{r 0} s_{z 0}}{4 A_{r} s_{z 0}+A_{z} s_{r 0}}\right)\right)^{-1}, \\
= & -4 \mu_{0} A_{r}\left(H_{c} h_{m}\right)^{2} l^{2} \\
& \times\left(s_{r 0}\left(\frac{4 A_{r} s_{z 0}+A_{z} s_{r 0}}{s_{z 0}}\right)^{2}\right. \\
& \left.\times\left(\frac{h_{m}}{\mu_{r} A_{m}}+\frac{2 s_{r 0} s_{z 0}}{4 A_{r} s_{z 0}+A_{z} s_{r 0}}\right)^{2}\right)^{-1} .
\end{aligned}
$$

\subsection{The Coupling Model of the Combined 5-DOFs HMB}

3.4.1. The Current Stiffness and Position of the RMB Unit Affected by the Axial Air Gap. The current stiffness $k_{r i}$ and position stiffness $k_{r s}$ of the RMB unit in (12) do not consider the effect of the rotor in $z$-axis direction, namely, the axial air gap. However, the bias flux generated from the PMR flows across the radial air gaps and the axial air gaps (Figure 5(a)); the alternation of the axial air gap will affect the current stiffness and the position stiffness of the RMB and will be calculated by

$$
\begin{aligned}
k_{r i}^{z}= & \left.\frac{\partial f_{A y}}{\partial i_{A y}}\right|_{\substack{i_{A y}=i_{z}=0 \\
x=y=0}} \\
= & 2 \mu_{0} A_{r} N_{r} H_{c} h_{m} \\
& \times\left(s_{r 0}^{2}\left(\frac{4 A_{r}}{s_{r 0}}+\frac{A_{z}}{s_{z 0}+z}\right)\right. \\
& \times\left(\frac{h_{m}}{\mu_{r} A_{m}}+\frac{\left(s_{z 0}+z\right) s_{r 0}}{4 A_{r}\left(s_{z 0}+z\right)+A_{z} s_{r 0}}\right.
\end{aligned}
$$




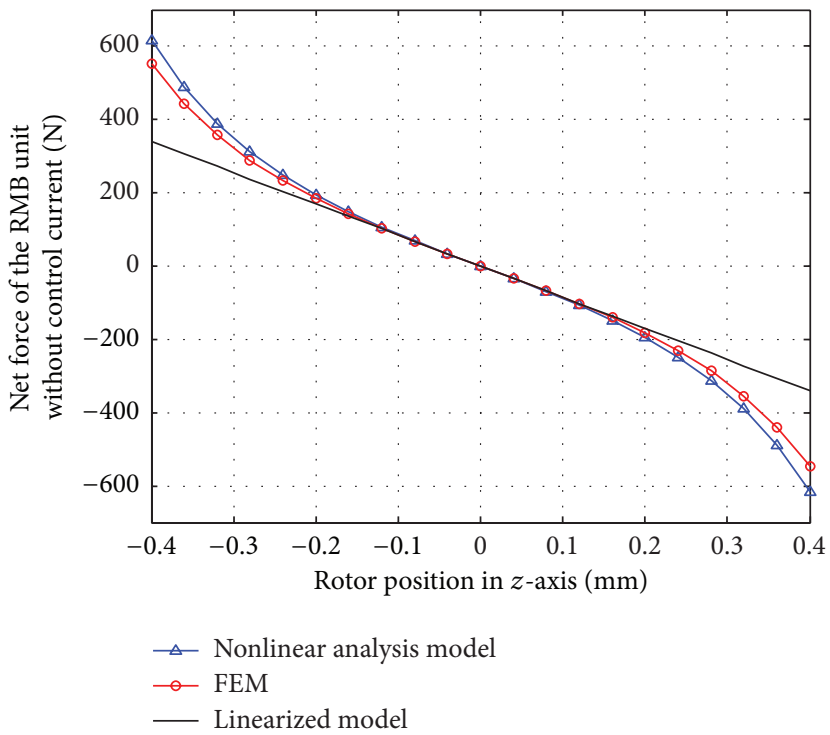

(a)

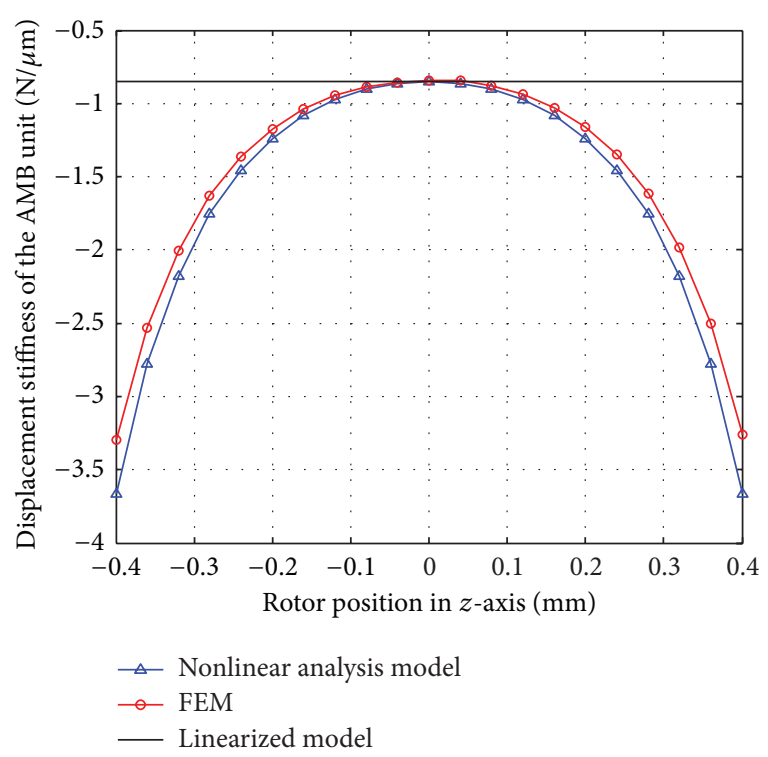

(b)

FIGURE 12: The force-rotor position in $z$-axis of the AMB unit and its displacement stiffness (using linearized method, nonlinear method, and FEM). (a) The force-rotor position in $z$-axis of the AMB unit. (b) The displacement stiffness of the AMB unit.

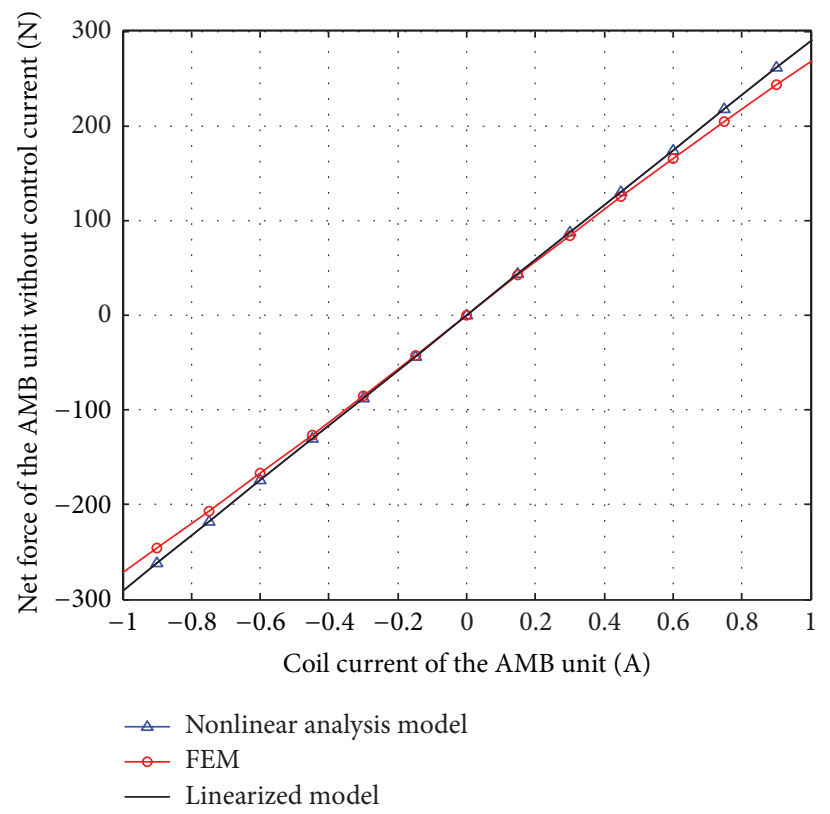

FIGURE 13: The force-coil current in $z$-axis of the AMB unit (using linearized method, nonlinear method, and FEM).

$$
\begin{gathered}
\left.\left.\quad+\frac{\left(s_{z 0}-z\right) s_{r 0}}{4 A_{r}\left(s_{z 0}-z\right)+A_{z} s_{r 0}}\right)\right)^{-1}, \\
k_{r s}^{z}=\left.\frac{\partial f_{A y}}{\partial y}\right|_{\substack{i_{A y}=i_{z}=0 \\
x=y=0}}=-2 \mu_{0} A_{r}\left(H_{c} h_{m}\right)^{2} \\
\quad \times\left(s_{r 0}^{3}\left(\frac{4 A_{r}}{s_{r 0}}+\frac{A_{z}}{s_{z 0}+z}\right)^{2}\right.
\end{gathered}
$$$$
\begin{gathered}
\times\left(\frac{h_{m}}{\mu_{r} A_{m}}+\frac{s_{r 0}\left(s_{z 0}+z\right)}{4 A_{r}\left(s_{z 0}+z\right)+A_{z} s_{r 0}}\right. \\
\left.\left.+\frac{s_{r 0}\left(s_{z 0}-z\right)}{4 A_{r}\left(s_{z 0}-z\right)+A_{z} s_{r 0}}\right)^{2}\right)^{-1}
\end{gathered}
$$

3.4.2. The Current Stiffness and Displacement Stiffness of the $R M B$ Unit Affected by the Coil Current in AMB Unit. From Figure $5(\mathrm{~d})$, the control flux generated by the coil current in 


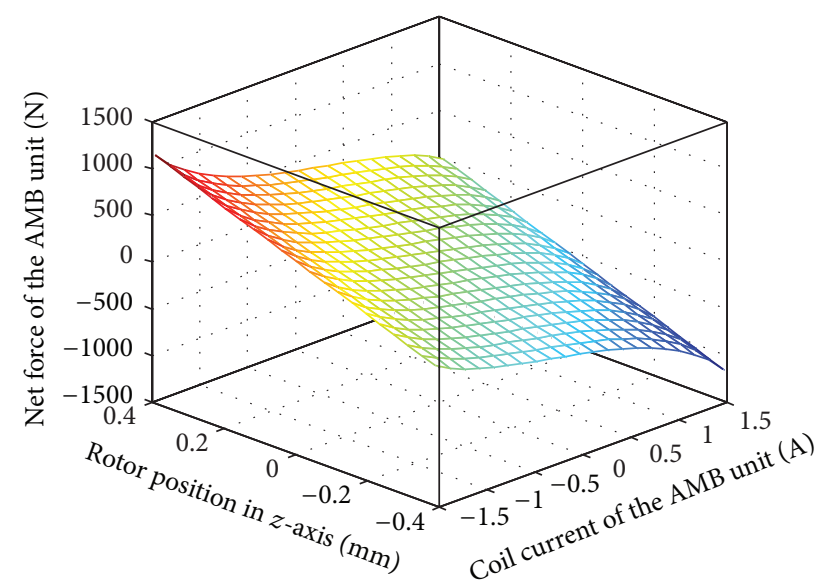

FIGURE 14: The force-coil current and rotor position in $z$-axis of the $\mathrm{AMB}$ unit (using nonlinear method).

AMB unit will flow through radial air gaps of the RMB unit, and the current stiffness and displacement stiffness of the $\mathrm{RMB}$ unit could be affected and given by

$$
\begin{aligned}
& k_{r i}^{i_{z}}=\left.\frac{\partial f_{A y}}{\partial i_{A y}}\right|_{\substack{i_{A y}=i_{B y}=0 \\
x=y=z=0}} \\
& =2 \mu_{0} A_{r} N_{r} H_{c} h_{m} \\
& \times\left(\left(4 A_{r} s_{r 0}+\frac{s_{r 0}^{2} A_{z}}{s_{z 0}}\right)\right. \\
& \left.\times\left(\frac{h_{m}}{\mu_{r} A_{m}}+\frac{2 s_{r 0} s_{z 0}}{4 A_{r} s_{z 0}+A_{z} s_{r 0}}\right)\right)^{-1} \\
& -\mu_{0} A_{r} N_{r} N_{z} i_{z} h_{m} \\
& \times\left(s_{r 0}\left(\frac{h_{m} s_{r 0}}{\mu_{0} A_{m} s_{r 0}+2 A_{r} h_{m}}+\frac{2 s_{z 0}}{A_{z}}\right)\right. \\
& \left.\times\left(\mu_{r} A_{m} s_{r 0}+2 A_{r} h_{m}\right)\right)^{-1}, \\
& k_{r s}^{i_{z}}=\left.\frac{\partial f_{A y}}{\partial y}\right|_{\substack{i_{A y}=i_{B y}=0 \\
x=y=z=0}} \\
& =-\frac{2 \mu_{0} A_{r}}{s_{r 0}}\left[H_{c} h_{m}\right. \\
& \times\left(\left(4 A_{r}+\frac{s_{r 0} A_{z}}{s_{z 0}}\right)\right. \\
& \left.\times\left(\frac{h_{m}}{\mu_{r} A_{m}}+\frac{2 s_{r 0} s_{z 0}}{4 A_{r} s_{z 0}+A_{z} s_{r 0}}\right)\right)^{-1} \\
& -N_{z} i_{z}\left(h_{m} s_{r 0}\right)
\end{aligned}
$$

$$
\begin{gathered}
\times\left(2 s_{r 0}\left(\frac{h_{m} s_{r 0}}{\mu_{0} A_{m} s_{r 0}+2 A_{r} h_{m}}+\frac{2 s_{z 0}}{A_{z}}\right)\right. \\
\left.\left.\times\left(\mu_{r} A_{m} s_{r 0}+2 A_{r} h_{m}\right)\right)^{-1}\right]^{2} .
\end{gathered}
$$

3.4.3. The Current Stiffness and Displacement Stiffness of the $A M B$ Unit Affected by the Radial Air Gap. The alternation of the radial air gap will affect the current stiffness and the displacement stiffness of the AMB unit and will be calculated by

$$
\begin{aligned}
& k_{z i}^{y}=\left.\frac{\partial f_{z}}{\partial i_{z}}\right|_{\substack{i_{A y}=i_{B y}=i_{z}=0 \\
x=z=0}} \\
& =2 \mu_{0} N_{z} H_{c} h_{m}\left(\frac{A_{r}}{s_{r 0}-y}+\frac{A_{r}}{s_{r 0}+y}+\frac{2 A_{r}}{s_{r 0}}+\frac{A_{z}}{s_{z 0}}\right) \\
& \times\left(s _ { z 0 } \left(\frac{h_{m}}{\mu_{r} A_{m}}\right.\right. \\
& \left.+\frac{2}{2 A_{r} s_{r 0} /\left(s_{r 0}^{2}-y^{2}\right)+2 A_{r} / s_{r 0}+A_{z} / s_{z 0}}\right) \\
& \times\left(\frac{1}{h_{m} / \mu_{r} A_{m}+2 A_{r} s_{r 0} /\left(s_{r 0}^{2}-y^{2}\right)+A_{r} / s_{r 0}}\right. \\
& \left.\left.+\frac{2 s_{z 0}}{A_{z}}\right)\right)^{-1} \\
& k_{z s}^{y}=\left.\frac{\partial f_{z}}{\partial z}\right|_{\substack{i_{A y}=i_{B y}=i_{z}=0 \\
x=z=0}} \\
& =\frac{2 \mu_{0} A_{z}\left(H_{c} h_{m}\right)^{2}}{\left(\left(h_{m} / \mu_{r} A_{m}\right)+\left(2 s_{r 0} s_{z 0} /\left(4 A_{r} s_{z 0}+A_{z} s_{r 0}\right)\right)\right)^{2}} \\
& \times \frac{A_{z}-s_{z 0}\left(\left(4 A_{r} / s_{r 0}\right)+\left(A_{z} / s_{z 0}\right)\right)}{s_{z 0}^{4}\left(\left(4 A_{r} / s_{r 0}\right)+\left(A_{z} / s_{z 0}\right)\right)^{3}} .
\end{aligned}
$$

\section{The Performance Analysis of the Combined 5-DOFs HMB Using Field-Circuit Method}

4.1. The Combined 5-DOFs HMB Parameters and the 3D Finite Element Model. The combined 5-DOFs HMB parameters are given in Table 1 . The example aims to validate the design and analysis method based on linearized model and 3D finite element method (FEM). 
TABLE 1: Design parameters of the combined 5-DOFs HMB.

\begin{tabular}{ll}
\hline Parameters & Value \\
\hline Inner diameter of the RMB unit rotor stacks $(\mathrm{mm})$ & 46 \\
Outer diameter of the RMB unit rotor stacks $(\mathrm{mm})$ & 68 \\
Length of the RMB unit rotor stacks (mm) & 20 \\
Inner diameter of the RMB unit stator (mm) & 69 \\
Outer diameter of the RMB unit stator (mm) & 113 \\
Length of the RMB unit stator (mm) & 20 \\
Radial pole shoe area (mm $\left.{ }^{2}\right)$ & 734 \\
The number of winding turns in coil of RMB unit & 220 \\
Inner diameter of the axial pole shoe (mm) & 76 \\
Outer diameter of the axial pole shoe (mm) & 90 \\
The number of winding turns in coil of AMB unit & 250 \\
The radial and axial air gap (mm) & 0.5 \\
Relative permeability of the permanent magnet ring & 1.082 \\
Outer diameter of the permanent magnet ring (mm) & 126 \\
Inner diameter of the permanent magnet ring (mm) & 113 \\
Length of the permanent magnet ring (mm) & 5 \\
\hline
\end{tabular}

The linearized model is given by

$$
\begin{aligned}
& f_{x}=k_{x i} i_{x}+k_{x s} x=284.4 i_{x}-5.5195 \times 10^{5} x, \\
& f_{y}=k_{y i} i_{y}+k_{y s} y=284.4 i_{y}-5.5195 \times 10^{5} y, \\
& f_{z}=k_{z i} i_{z}+k_{z s} z=290.5 i_{z}-8.5007 \times 10^{5} z, \\
& M_{x}=k_{\alpha i} i_{\alpha}+k_{\alpha s} \alpha=39.25 i_{\alpha}-5255.6 \alpha, \\
& M_{y}=k_{\beta i} i_{\beta}+k_{\beta s} \beta=39.25 i_{\beta}-5255.6 \beta,
\end{aligned}
$$

where $k_{i x}$ and $k_{i y}$ are the current stiffness of the system in $x$ axis and $y$-axis, respectively; $k_{s p}$ is the displacement stiffness of the system in $x$-axis and $y$-axis; $k_{z}$ is the displacement stiffness in $z$-axis; $k_{\alpha}$ and $k_{\beta}$ are the tilting displacement stiffness around $x$-axis and $y$-axis; $k_{\alpha i}$ and $k_{\beta i}$ are the tilting current stiffness around $x$-axis and $y$-axis. Moreover, the rotor can be controlled in five degrees-of-freedom.

The finite element model using Ansoft Maxwell V13 is built and shown in Figure 6(a) except the air. Its meshes are shown in Figures 6(b) and 6(c), and the total number of mesh is 286036 .

\subsection{The Performance Analysis for Radial and Axial \\ Translation of the Combined 5-DOFs HMB}

4.2.1. The Analysis Results of the Force-Displacement and Displacement Stiffness of the RMB Unit (Using Linearized Method, Nonlinear Method, and FEM). When the control currents in radial coil currents and axial coil current are $0 \mathrm{~A}$, and the rotor position changes from $-0.4 \mathrm{~mm}$ to $0.4 \mathrm{~mm}$ in $x$-axis (or $y$-axis), and hold in center position in $z$-axial direction, the relationship between net force and the rotor position in $x$-axis (or $y$-axis) direction is shown in Figure 7 (a) using linearized method, nonlinear method, and FEM. It is obvious that the force is nonlinear. The calculated results are approximately equal for small values of the rotor in $x$ axis (or $y$-axis) and there is little difference between the nonlinear model and FEM. In addition, the displacement stiffnesses using the three calculated methods are shown in Figure 7(b). The displacement stiffness in rotor center is $-5.5195 \times 10^{5} \mathrm{~N} / \mathrm{m}$ using linearized method and nonlinear method, and the displacement stiffness calculated by the FEM in rotor center is $-5.3815 \times 10^{5} \mathrm{~N} / \mathrm{m}$; the calculated error between them is $2.6 \%$.

4.2.2. The Analysis Results of the Force-Coil Current and Current Stiffness of the RMB Unit (Using Linearized Method, Nonlinear Method, and FEM). When the rotor locates at radial and axial center and the coil current in $x$-axis or $y$ axis of the $\mathrm{RMB}$ unit changes from $-1 \mathrm{~A}$ to $1 \mathrm{~A}$ (the coil current of the AMB unit is $0 \mathrm{~A}$ ), the relationship between net force of the RMB unit and coil current is shown in Figure 8 using linearized method (see (11)), nonlinear method (see (1)), and FEM. It is obvious that the net force is linear, and the calculated results are approximately equal. In addition, the current stiffness of the RMB unit using the three calculated methods is $284.4 \mathrm{~N} / \mathrm{A}$ (linearized method and nonlinear method) and $284.13 \mathrm{~N} / \mathrm{A}$ (FEM); the calculated error is between linearized method and FEM 1.5\%.

4.2.3. The Analysis Results of the Force-Coil Current and Rotor Position in $x$-Axis (or $y$-Axis) of the RMB Unit (Using Nonlinear Method). When the rotor in $z$-axis locates its center position and the coil current of the AMB unit is $0 \mathrm{~A}$, the curves of the net force-coil current-rotor position of the RMB unit in $x$-axis (or $y$-axis) using nonlinear method are shown in Figure 9. It is obvious that it is approximately linearity for small displacement and coil current and nonlinear for large displacement and coil current. The displacement stiffness and current stiffness affected by the rotor position and the coil current in $x$-axis (or $y$-axis) of the RMB unit are shown in Figures 10 and 11, respectively. They are approximately linear for small displacement and coil current.

4.2.4. The Analysis Results of the Force-Displacement and Displacement Stiffness of the AMB Unit (Using Linearized Method, Nonlinear Method, and FEM). When the control currents in radial coil currents and axial coil current all are $0 \mathrm{~A}$, and the rotor position changes from $-0.4 \mathrm{~mm}$ to $0.4 \mathrm{~mm}$ in $z$-axis, and hold in center position in $x$-axial and $y$-axis direction, the relationship between net force and the rotor position in $z$-axis direction is shown in Figure 12(a) using linearized method (see (11)), nonlinear method (see (1)), and FEM. It is obvious that the force is also nonlinear. The calculated results are approximately equal for small values of the rotor in $z$-axis and there is little difference between the nonlinear model and FEM. In addition, the displacement stiffness using the three calculated methods is shown in Figure 12(b). The calculated results are approximately equal for small value of the displacement in $z$-axis between the linearized method and FEM. The displacement stiffness in rotor center is $-8.5007 \times 10^{5} \mathrm{~N} / \mathrm{m}$ using linearized method and nonlinear method, and the displacement stiffness calculated by the FEM 


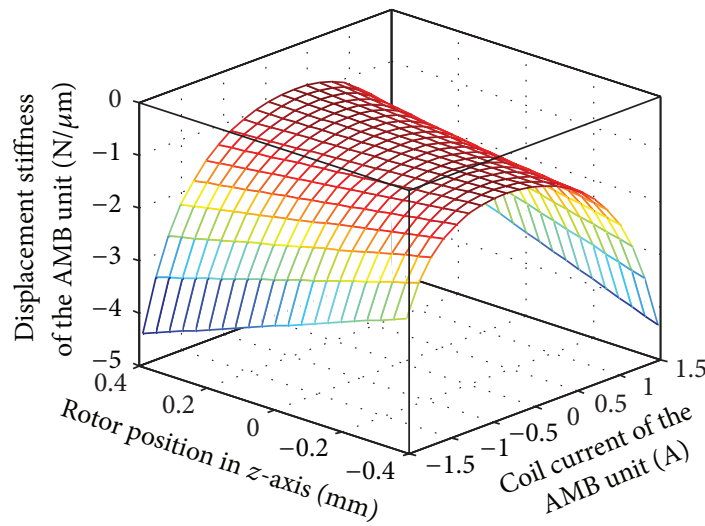

(a)

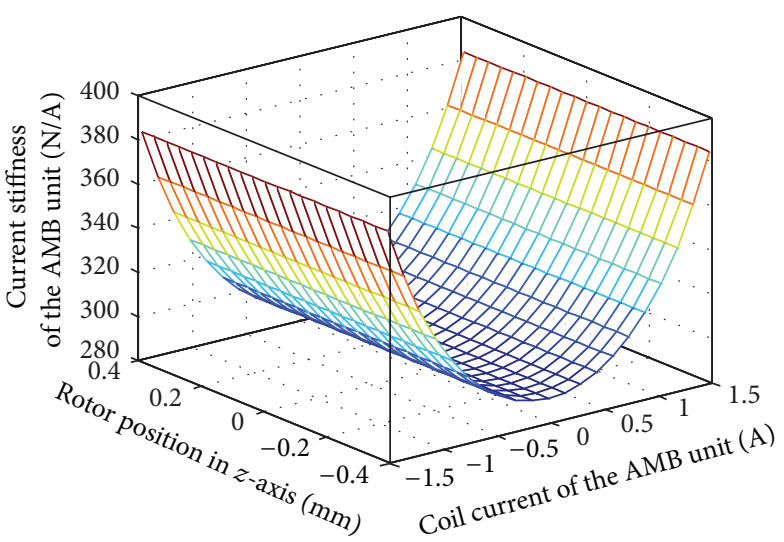

(b)

Figure 15: The curves of the stiffness versus rotor position in $z$-axis and coil current of the AMB unit (using nonlinear method). (a) The displacement stiffness versus coil current and rotor position in $z$-axis of the AMB unit. (b) The current stiffness versus coil current and rotor position in $z$-axis of the AMB unit.

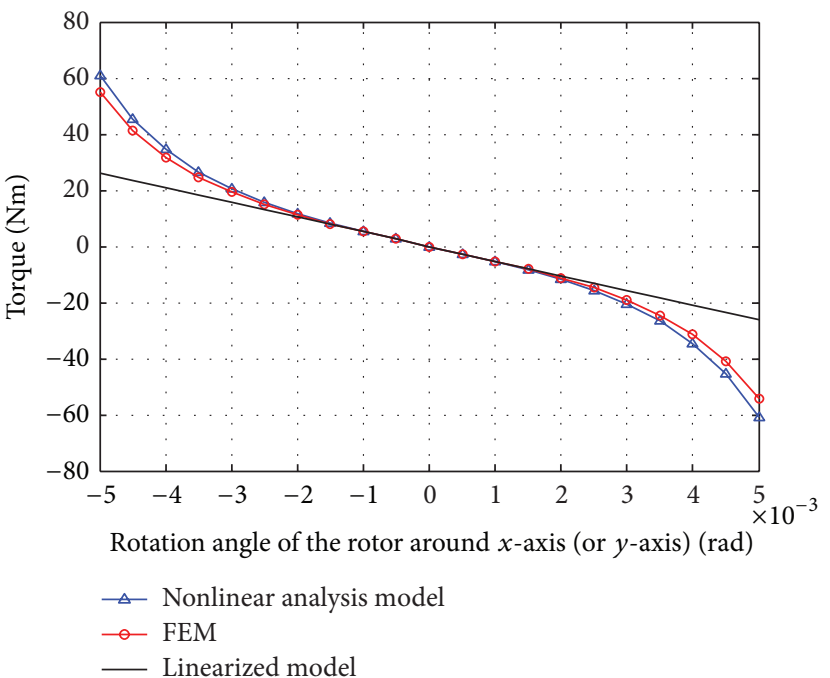

(a)

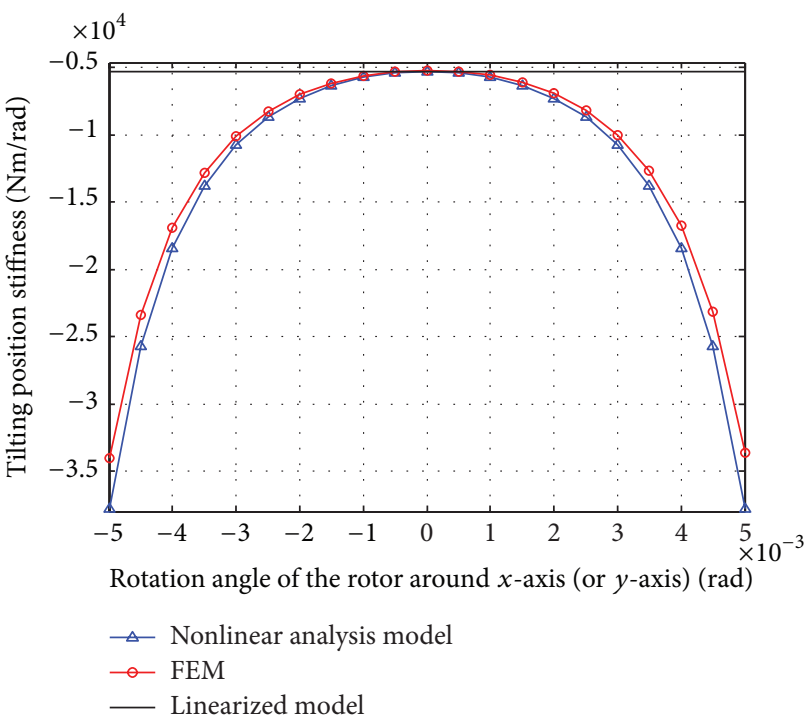

(b)

FIGURE 16: Curves of the torque-angular position of the combined 5-DOFs HMB and its tilting displacement stiffness (using linearized method, nonlinear method, and FEM). (a) Curves of the torque-angular position of the combined 5-DOFs HMB. (b) The tilting displacement stiffness.

in rotor center is $-8.3732 \times 10^{5} \mathrm{~N} / \mathrm{m}$; the calculated error between them is $4.4 \%$.

4.2.5. The Analysis Results of the Force-Coil Current and Current Stiffness of the AMB Unit (Using Linearized Method, Nonlinear Method, and FEM). When the rotor locates at radial and axial center and the coil current in $z$-axis of the $\mathrm{AMB}$ unit changes from $-1 \mathrm{~A}$ to $1 \mathrm{~A}$ (the coil currents of the $\mathrm{RMB}$ unit are $0 \mathrm{~A}$ ), the relationship between net force of $\mathrm{AMB}$ unit and its coil current is shown in Figure 13 using linearized method (see (15)), nonlinear method (see (13)), and FEM.
It is obvious that the net force is linear, and the calculated results are approximately equal. In addition, the current stiffness of the AMB unit using the three calculated methods is $290.5 \mathrm{~N} / \mathrm{A}$ (linearized method and nonlinear method) and $280.3 \mathrm{~N} / \mathrm{A}$ (FEM); the calculated error is $3.5 \%$ between linearized method and FEM.

4.2.6. The Analysis Results of the Force-Coil Current and Rotor Position in $z$-Axis of the AMB Unit (Using Nonlinear Method). When the rotor locates its center position in $x$ axis and $y$-axis and the coil currents of the RMB units are 
$0 \mathrm{~A}$, the curves of the net force-coil current-rotor position of the AMB unit in $z$-axis using nonlinear method are shown in Figure 14. It is obvious that the relationship of the net force-displacement in $z$-axis is approximately linear and the relationship of the net force-versus coil current is approximately linear for small displacement and nonlinear for large coil current. The displacement stiffness affected by the rotor position and the coil current in $z$-axis of the AMB unit is shown in Figure 15(a). The current stiffness affected by the rotor position and the coil current in $z$-axis of the AMB unit is shown in Figure 15(b). In addition, the current stiffness is only affected by the coil current of the AMB unit.

\subsection{The Performance Analysis for Tilting of the Combined 5-DOFs $H M B$}

4.3.1. The Analysis Results of the Torque-Angular Position and Angular Displacement Stiffness of the Combined 5-DOFs $H M B$. The torque can be produced by the RMB units $A$ and $B$. When the control currents in radial coil and axial coil all are $0 \mathrm{~A}$ and the rotor tilts within $-5 \mathrm{mrad} \sim 5 \mathrm{mrad}$ around $x$ axis or $y$-axis, the relationship between torque and angular position of rotor is shown in Figure 16(a) using linearized method, nonlinear method, and FEM. The calculated results are approximately equal for small values of the angular position of rotor and there is little difference between the nonlinear model and FEM. In addition, the tilting position stiffness is shown in Figure 16(b). The calculated results are approximately equal for small value of the angular position between the linearized method and FEM. The angular displacement stiffness in rotor center is $-5255.6 \mathrm{Nm} / \mathrm{rad}$.

4.3.2. The Analysis Results of the Torque-Coil Current and Tilting Current Stiffness of the Combined 5-DOFs HMB (Using Linearized Method, Nonlinear Method, and FEM). When the rotor locates at radial and axial center and the coil currents of the RMB units $A$ and $B$ in $x$-axis or $y$-axis change from $-1 \mathrm{~A}$ to $1 \mathrm{~A}$ (the coil current of $\mathrm{AMB}$ unit is $0 \mathrm{~A}$ ), the relationship between torque and coil current of the RMB units is shown in Figure 17 using linearized method (see (15)), nonlinear method (see (13)) and FEM. It is obvious that the torque is linear, and the calculated results are approximately equal. In addition, the tilting current stiffness using the three calculated methods is $39.25 \mathrm{Nm} / \mathrm{A}$ (linearized method and nonlinear method) and $38.07 \mathrm{Nm} / \mathrm{A}$ (FEM); the calculated error between the linearized method and FEM is 3\%.

4.3.3. The Analysis Results of the Torque-Coil Current and Angular Position in $x$-Axis or $y$-Axis of the Combined 5-DOFs $H M B$ (Using Linearized Method and Nonlinear Method). When the rotor locates its center position in $z$-axis and the coil currents of the AMB unit are $0 \mathrm{~A}$, the curves of the torque produced by the RMB units $A$ and $B$ versus their coil current and rotor angular position around $x$-axis or $y$ axis using nonlinear method are shown in Figure 18 using linearized method and nonlinear method. It is obvious that it is approximately linear for small values of the angular position and coil current. Moreover, the curves of the angular

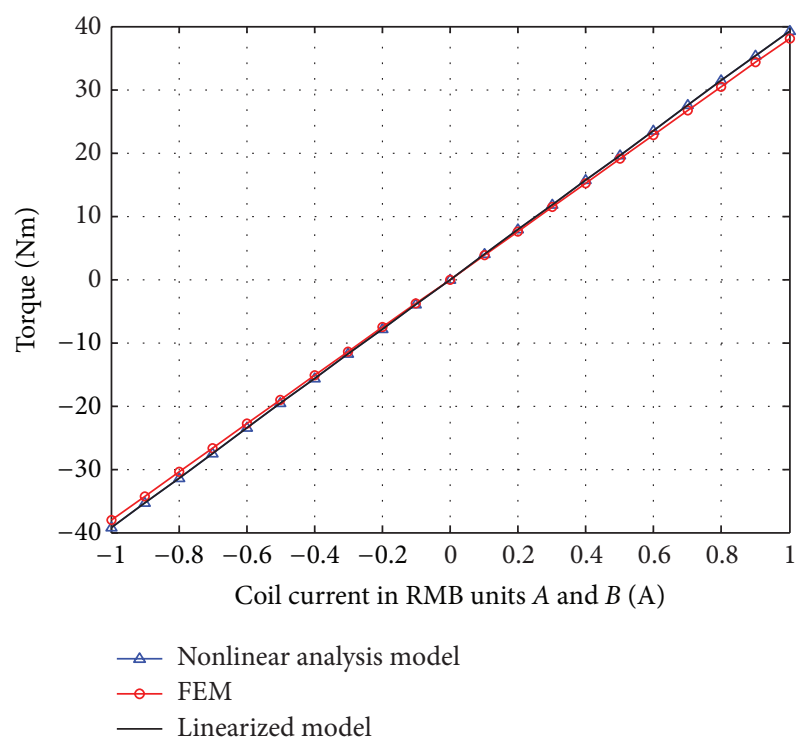

FIgURE 17: Curves of the torque-coil current in RMB units $A$ and $B$ of the combined 5-DOFs HMB (using linearized method, nonlinear method, and FEM).

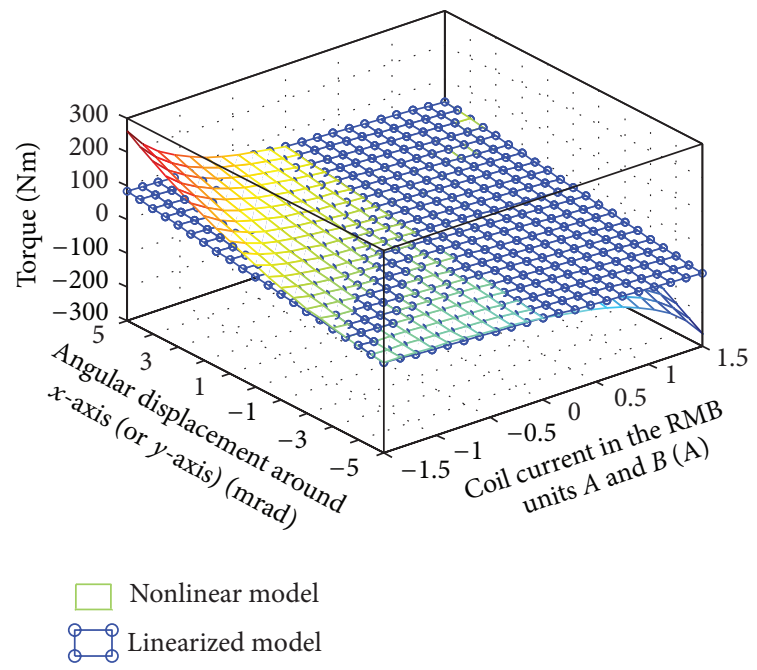

FIGURE 18: Curves of the torque-coil current and angular position around $x$-axis or $y$-axis in the RMB units $A$ and $B$ of the combined 5-DOFs HMB (using linearized method and nonlinear method).

position stiffness versus angular position and coil currents in RMB units $A$ and $B$ are shown in Figure 19(a). And the curves of the tilting current stiffness versus angular position and coil currents in RMB units $A$ and $B$ are shown in Figure 19(b). They all are affected by the angular position and coil currents in RMB units $A$ and $B$.

\subsection{The Coupling Performance Analysis of the Combined 5-DOFs $H M B$}

4.4.1. The Coupling Performance Analysis for Translation of the Combined 5-DOFs HMB. Equation (22) shows that 


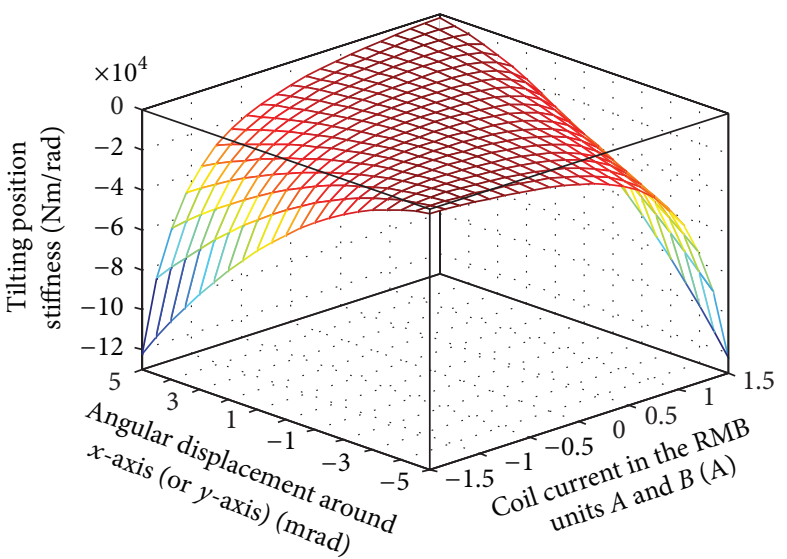

(a)

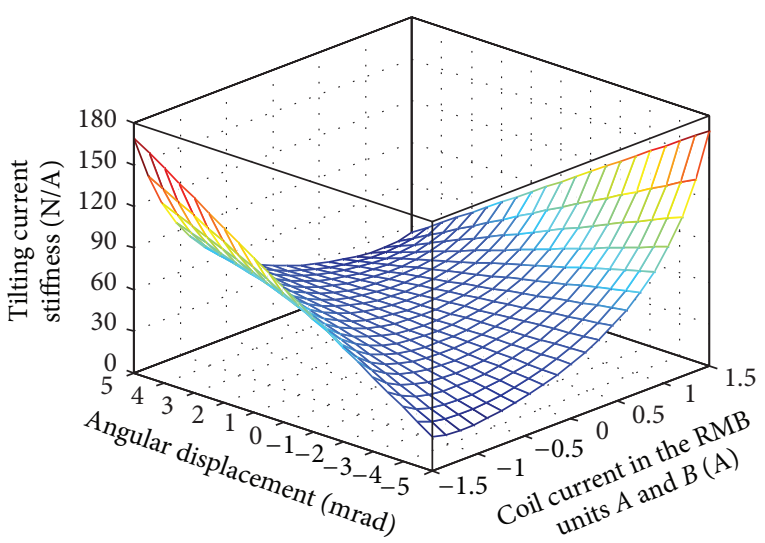

(b)

FIGURE 19: Curves of the tilting displacement stiffness and the tilting current stiffness versus angular position around $x$-axis or $y$-axis and the coil current in the RMB units $A$ and $B$ of the combined 5-DOFs HMB (using linearized method and nonlinear method). (a) Tilting displacement stiffness versus angular position around $x$-axis or $y$-axis and the coil current. (b) Tilting current stiffness versus angular position around $x$-axis or $y$-axis and the coil current.

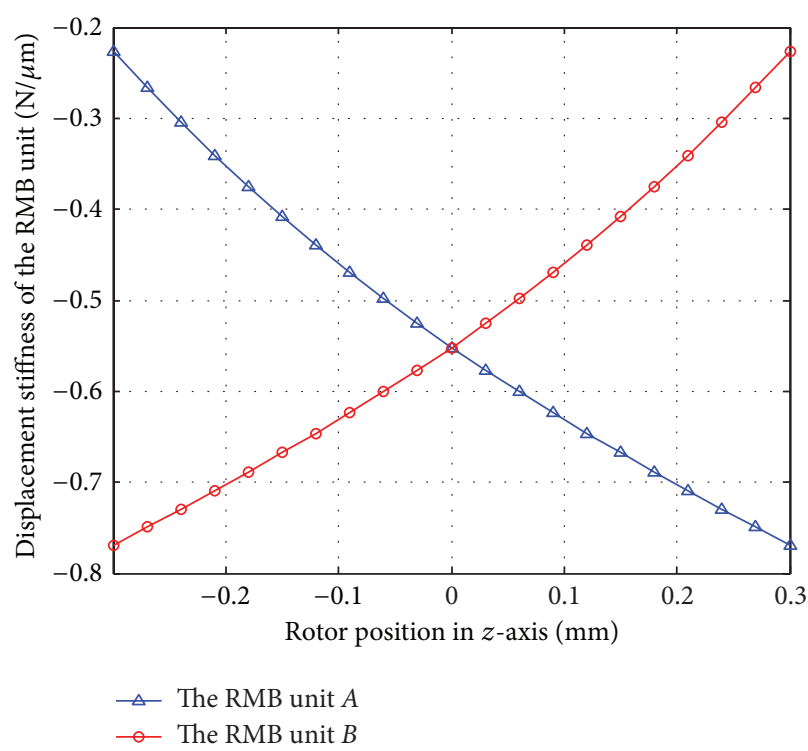

(a)

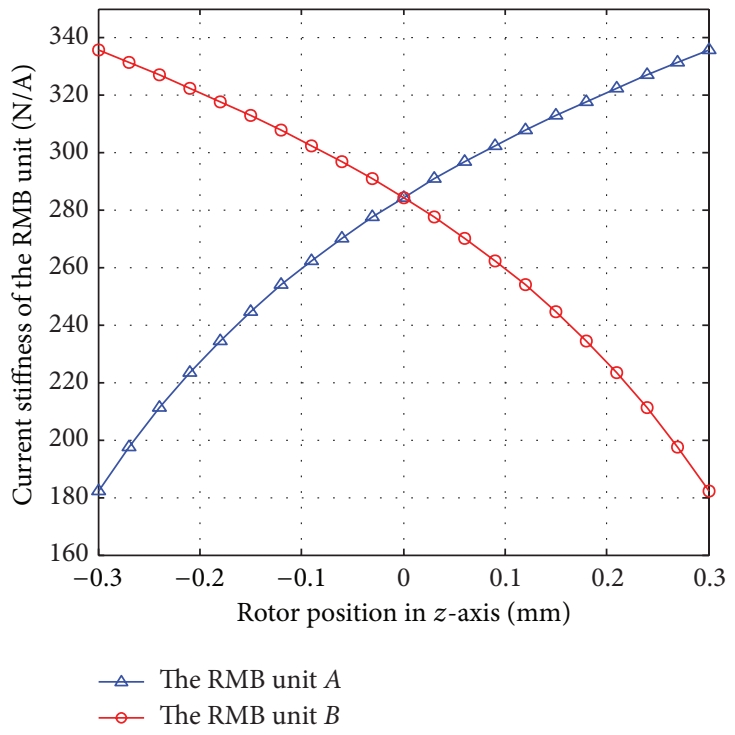

(b)

FIgUre 20: The displacement stiffness and the current stiffness of the RMB units $A$ and $B$ affected by the rotor position of the AMB unit in $z$-axis. (a) The displacement stiffness of the RMB units $A$ and $B$ affected by the rotor position of the AMB unit in $z$-axis. (b) The current stiffness of the RMB units $A$ and $B$ affected by the rotor position of the AMB unit in $z$-axis.

the displacement stiffness and current stiffness of the RMB unit will be affected by the rotor position in $z$-axis and the coil current of the AMB unit. The effect of the rotor position in $z$-axis on the displacement stiffness and current stiffness of the RMB units $A$ and $B$ is shown in Figures 20(a) and 20(b), respectively. They all are affected by the rotor position in $z$ axis and their change trend is opposite.

The effect of the coil current of the AMB unit on the displacement stiffness and current stiffness of the RMB units $A$ and $B$ is shown in Figures 21(a) and 21(b), respectively. They all are affected by the coil current of the AMB unit and their change trend is the same.

The 3D curves of the displacement stiffness and current stiffness of the RMB units $A$ and $B$ affected by the rotor position in $z$-axis and the coil current of the AMB unit are shown in Figures 22(a) and 22(b).

Figure 23 shows that the displacement stiffness and current stiffness of the AMB unit will be affected by the rotor position in $x$-axis or $y$-axis of the RMB unit. The effect of the rotor position in $x$-axis or $y$-axis on the displacement 


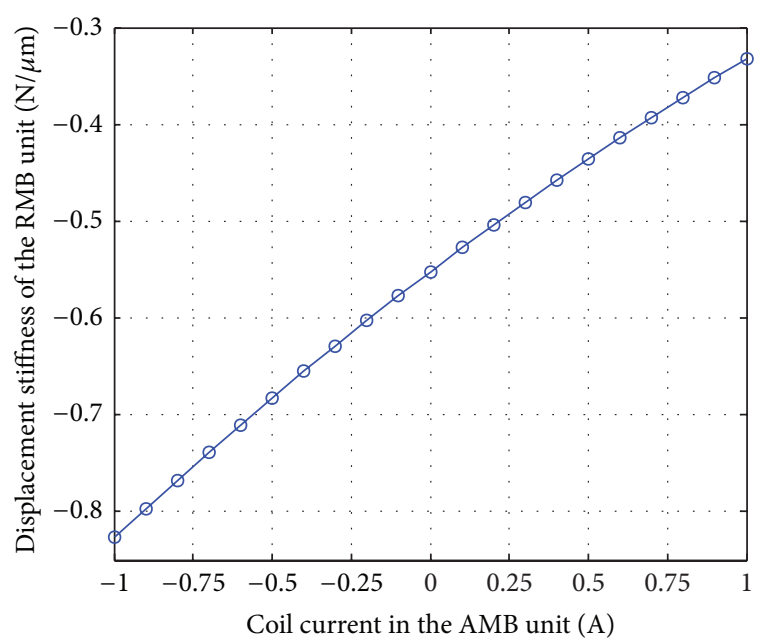

(a)

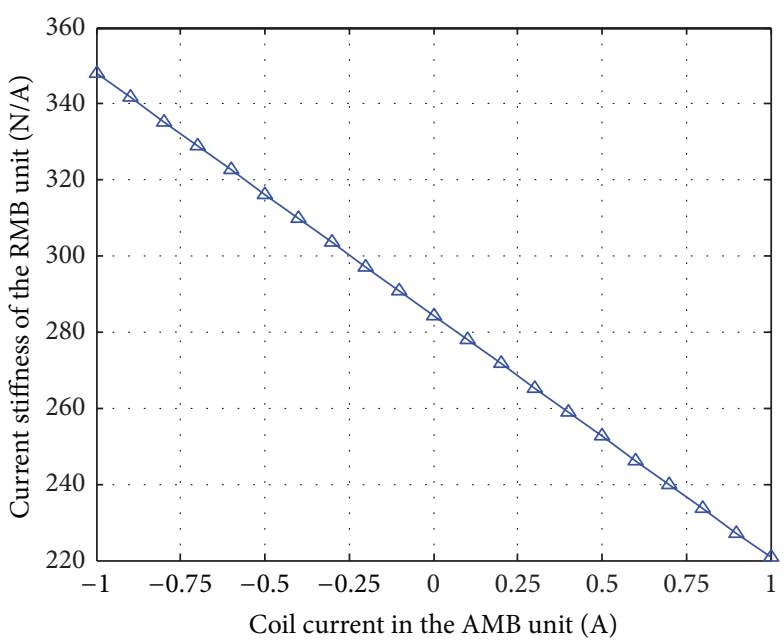

(b)

Figure 21: The displacement and current stiffness of the RMB units $A$ and $B$ affected by the coil current of the AMB unit. (a) The displacement stiffness of the RMB units $A$ and $B$ affected by the coil current of the AMB unit. (b) The current stiffness of the RMB units $A$ and $B$ affected by the coil current of the AMB unit.

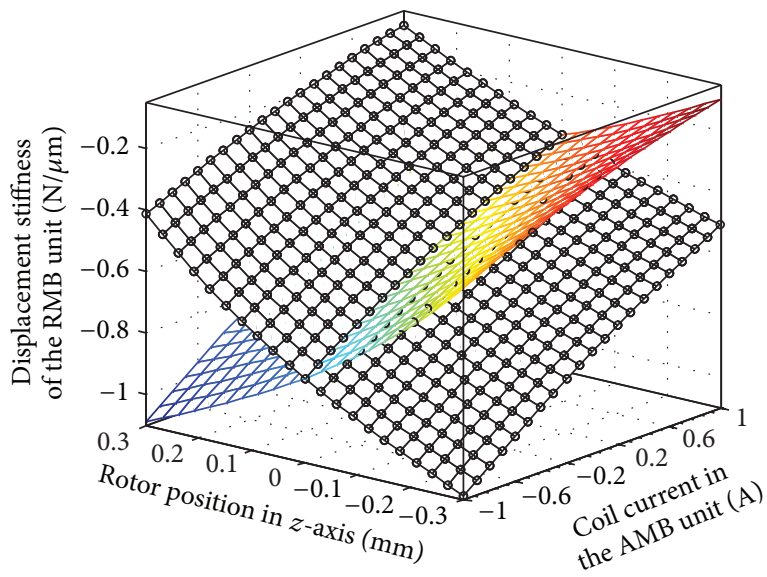

The RMB unit $A$

QS The RMB unit $B$

(a)

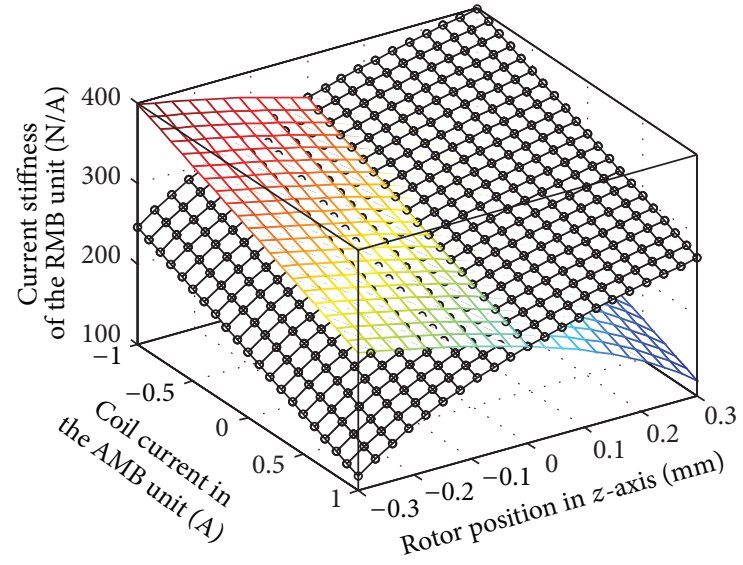

QS The RMB unit $A$

The RMB unit $B$

(b)

FIGURE 22: The displacement stiffness and the current stiffness of the RMB units $A$ and $B$ affected by the rotor position in $z$-axis and the coil current of the AMB unit. (a) The displacement stiffness of the RMB units $A$ and $B$ affected by the rotor position in $z$-axis and the coil current of the AMB unit. (b) The current stiffness of the RMB units $A$ and $B$ affected by the rotor position in $z$-axis and the coil current of the AMB unit.

stiffness and current stiffness of the AMB unit is shown in Figures 23(a) and 23(b), respectively. They all are affected by the rotor position in $x$-axis or $y$-axis.

4.4.2. The Coupling Performance Analysis for Tilting of the Combined 5-DOFs $H M B$. The tilting displacement stiffness and tilting current stiffness of the RMB units $A$ and $B$ will be affected by the rotor position in $z$-axis and coil current of the $\mathrm{AMB}$ unit, and the influence curves of RMB units $A$ and $B$ are shown in Figures 24(a), 24(b), 25(a), and 25(b), respectively. It is obvious that the tilting displacement stiffness and tilting current stiffness of the RMB units $A$ and $B$ all are affected by the rotor position in $z$-axis and coil current of the AMB unit.

\section{Conclusion}

A novel combined 5-DOFs HMB with only one PMR is proposed, used for turboexpander, in this paper. The combined 


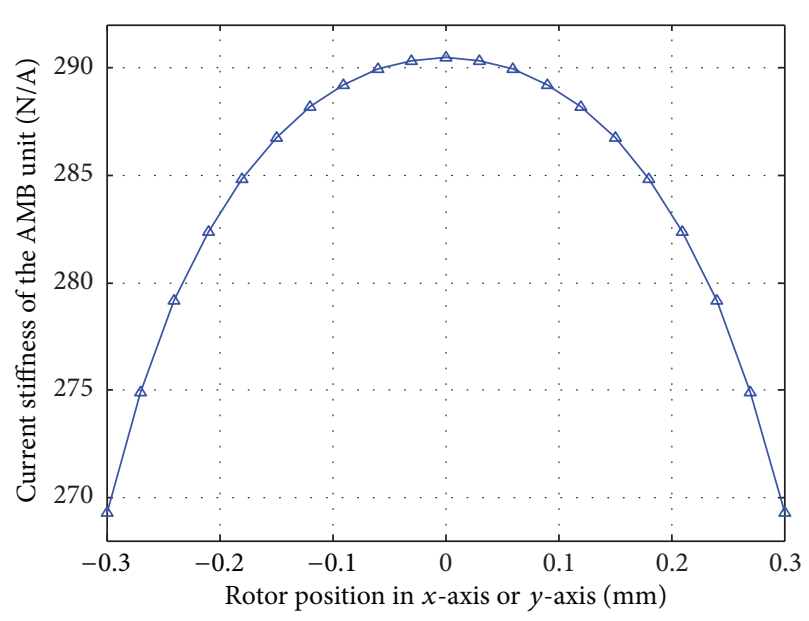

(a)

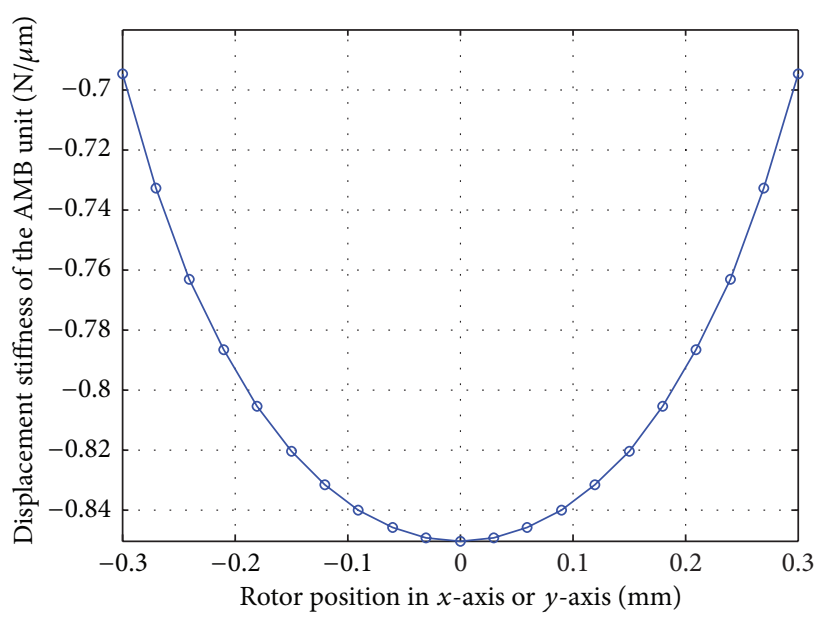

(b)

FIGURE 23: The displacement and the current stiffness of the AMB unit affected by the rotor position of the RMB unit in $x$-axis or $y$-axis. (a) The displacement stiffness of the AMB unit affected by the rotor position of the RMB unit. (b) The current stiffness of the AMB unit affected by the rotor position of the RMB unit.

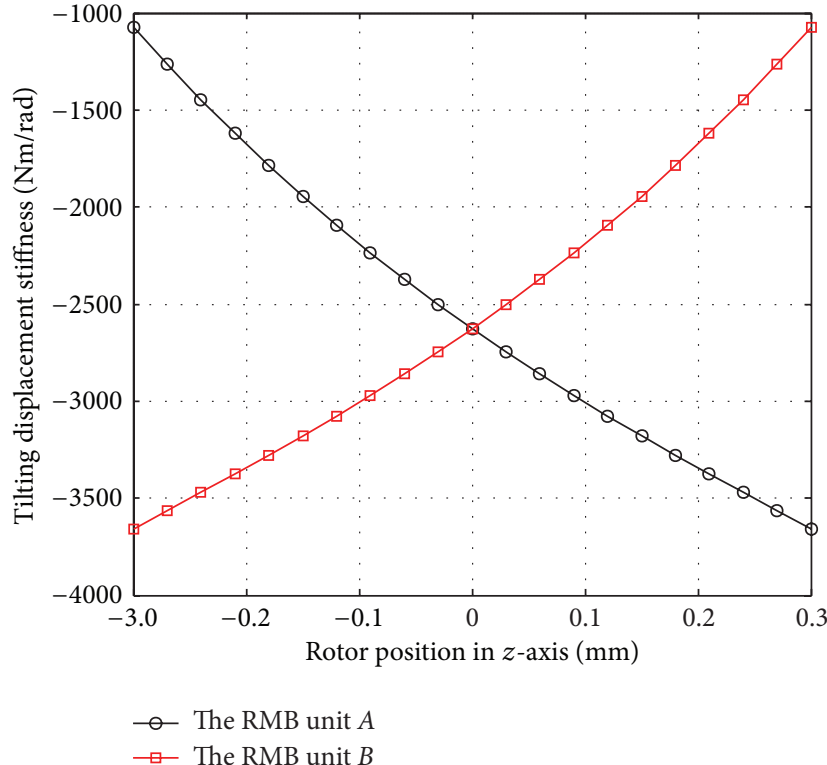

(a)

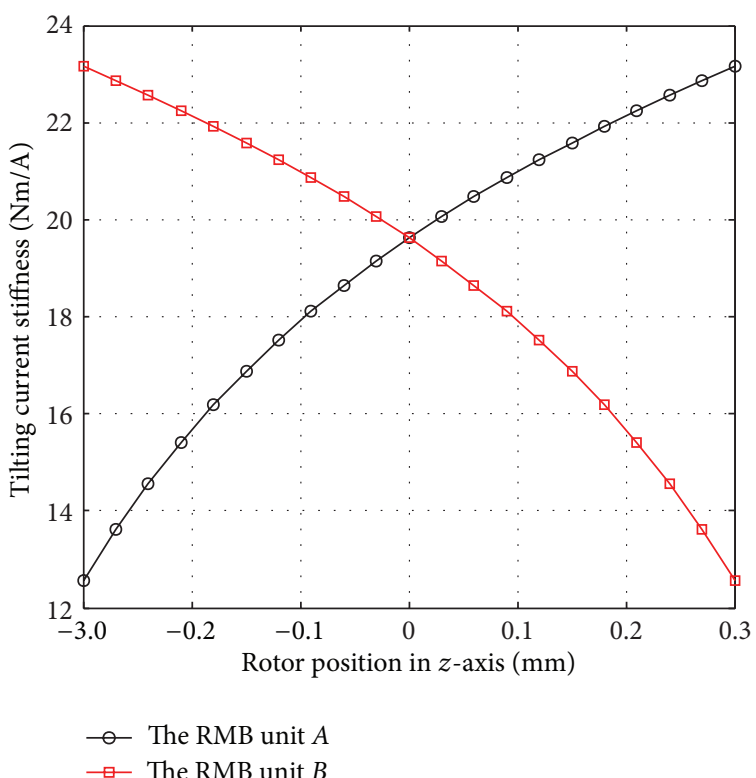

(b)

FIgURE 24: Curves of the tilting displacement stiffness and tilting current stiffness of the RMB units $A$ and $B$ affected by the rotor position in $z$-axis of the AMB unit. (a) The tilting displacement stiffness of the RMB units $A$ and $B$ affected by the rotor position in $z$-axis. (b) The tilting current stiffness of the RMB units $A$ and $B$ affected by the rotor position in $z$-axis of the AMB unit.

5-DOFs HMB consists of two RMB units and one AMB unit. The bias flux of the two RMB units and AMB unit is supplied by only one PMR. The low power consumption, compact structure, and small size can be gained due to the combined 5-DOFs HMB, the permanent magnetic bias HMB, and the reduction in the number of the poles of the RMB. The low cost is gained due to the one PMR used. Moreover, ultra-high-speed is also gained due to the small outside diameter of the rotor thrust disk. The equivalent magnetic circuit method and 3D finite element method (FEM) are used to model and analyze the integral 5-DOFs HMB. The forcecurrent, force-position, torque-coil currents, torque-angular position, and the stiffness models of the integral 5-DOFs $\mathrm{HMB}$ are given. Moreover, its coupling problems between the $\mathrm{RMB}$ units and the AMB unit are also proposed. An example is given to clarify the mathematical models and the coupling 


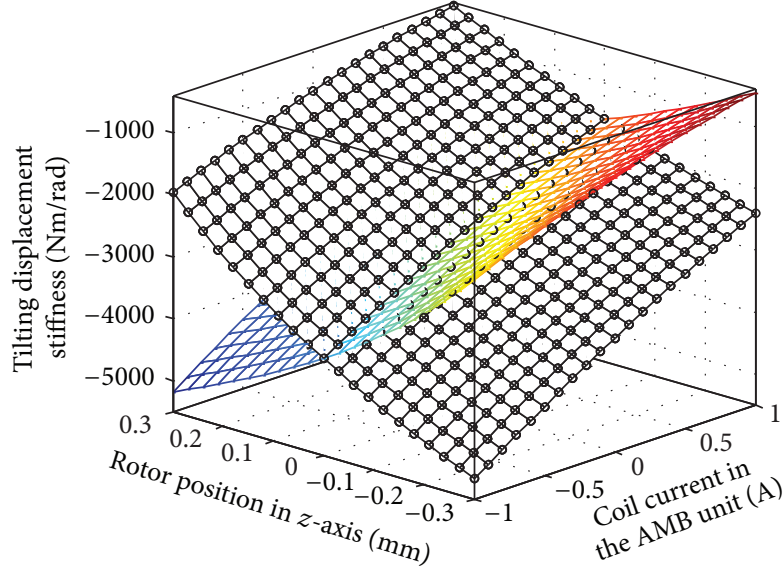

$\square$ The RMB unit $A$ \& The RMB unit $B$

(a)

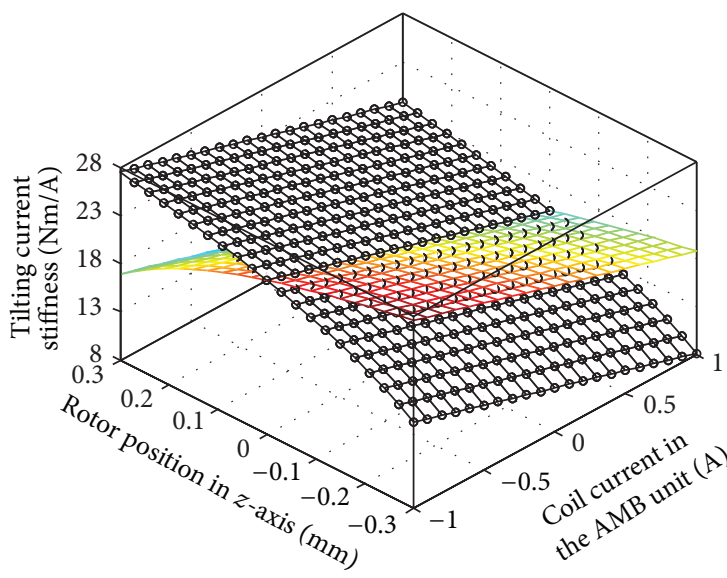

88 The RMB unit $A$ The RMB unit $B$

(b)

FIgURE 25: Curves of the tilting displacement stiffness and tilting current stiffness of the RMB units $A$ and $B$ affected by the rotor position in $z$-axis and coil current of the AMB unit. (a) Curves of the tilting displacement stiffness of the RMB units $A$ and $B$ affected by the rotor position in $z$-axis and coil current of the AMB unit. (b) Curves of the tilting current stiffness of the RMB units $A$ and $B$ affected by the rotor position in $z$-axis and coil current of the AMB unit.

problems, and the linearized models are proposed for the follow-up controller design.

\section{Conflict of Interests}

The authors declare no conflict of interests.

\section{Acknowledgments}

This work was supported by the Aviation Science Fund of China under Grant 2012ZB51019 and by the Cultivation and Development Project of Science and Technology Innovation Base of Beijing under Grant Z131104002813105.

\section{References}

[1] http://www.magnetic-bearings-s2m.com.

[2] http://www.ge-energy.com/about/oil_and_gas.jsp.

[3] http://www.skf.com/au/products/magnetic-systems/magneticsystems-applications/oil-gas/turboexpanders/index.html.

[4] J. Jumonville, "Tutorial on cryogenic turboexpanders," in Proceedings of the 33rd Turbomachinery Symposium, pp. 127-134, George R. Brown Convention Center, Houston, Tex, USA, September 2004.

[5] J. Jumonville, C. M. Ramsey, and F. Andrews, "Specifying, manufacturing, and testing a cryogenic turboexpander magnetic bearing system," in Proceedings of the 20th Turbomachinery Symposium, pp. 3-9, Turbomachinery Laboratory, Texas A\&M University, College Station, Tex, USA, 1991.

[6] H. Hirai, M. Hirokawa, S. Yoshida et al., "Development of a neon cryogenic turbo-expander with magnetic bearings," in Proceedings of the Transactions of the Cryogenic Engineering Conference-CEC: Advances in Cryogenic Engineering, vol. 1218 of AIP Proceedings, pp. 895-902, Tucson, Ariz, USA, 2010.
[7] W. Xiping, Z. Zhiming, Y. Liang, C. Weidong, and W. Jingui, "Experimental study of active magnetic bearing on a $150 \mathrm{M}^{3}$ turbo oxygen gas expander," Journal of Shanghai University, vol. 2, no. 4, pp. 334-336, 1998.

[8] W. Xiping, Z. Zhimin, Y. Liang, and W. Jingui, "Development of applied research of active magnetic bearing on a gas expander," China Mechanical Engineering, vol. 11, no. 4, pp. 379-381, 2000 (Chinese).

[9] M. Brune and I. Detomb, "Application of active magnetic bearings in turbocompressors and turboexpanders of the gas industry," Chemical and Petroleum Engineering, vol. 38, no. 7-8, pp. 459-463, 2002.

[10] W. R. Canders, N. Ueffing, U. Schrader-Hausm, and R. Larsonneur, "MTG400: a magnetically levitated $400 \mathrm{~kW}$ turbo generator system for natural gas expansion," in Proceedings of the 4th International Symposium on Magnetic Bearings, G. Schweitzer, Ed., pp. 435-440, ETH Zürich, Zürich, Switzerland, 1994.

[11] J. Schmied, "Rotordynamic aspects of a new hermetically sealed pipeline compressor," in Proceedings of the 20th Trubomachinery Symposium, pp. 11-17, Turbomachinery Laboratory, Texas A\&M University, College Station, Tex, USA, 1991.

[12] T. M. Brian, O. Hamid, T. C. Matthew, and J. D. Herbst, "Permanent magnet bias, homopolar magnetic bearings for a $130 \mathrm{~kW}$-hr composite flywheel," in Proceedings of the 19th International Symposium on Magnetic Bearings, pp. 66-72, Lexingtong, Ky, USA, 2004.

[13] Y. Zhilichev, "Analysis of a magnetic bearing pair with a permanent magnet excitation," IEEE Transactions on Magnetics, vol. 36, no. 5, pp. 3690-3692, 2000.

[14] C. Ehmann, T. Sielaff, and R. Nordmann, "Parison of active magnetic bearings with and without permanent magnet bias," in Proceedings of the 19th International Symposium on Magnetic Bearings, Lexington, Ky, USA, August 2004. 
[15] B. Han, S. Zheng, and H. Xiaofei, "Dynamic factor models of a thrust magnetic bearing with permanent magnet bias and subsidiary air gap," IEEE Transactions on Magnetics, vol. 49, no. 3, pp. 1221-1230, 2013.

[16] J. Fang and Y. Ren, "High-precision control for a single-gimbal magnetically suspended control moment gyro based on inverse system method," IEEE Transactions on Industrial Electronics, vol. 58, no. 9, pp. 4331-4342, 2011.

[17] C. H. Park, S. K. Choi, and S. Y. Ham, "Design and control for hybrid magnetic thrust bearing for turbo refrigerant compressor," in Proceedings of the 7th IEEE International Conference on Automation Science and Engineering (CASE '11), pp. 792-797, Trieste, Italy, August 2011.

[18] K.-C. Lee, Y.-H. Jeong, D.-H. Koo, and H. J. Ahn, "Development of a radial active magnetic bearing for high speed turbomachinery motors," in Proceedings of the SICE-ICASE International Joint Conference, pp. 1543-1548, Busan, South Korea, October 2006.

[19] J. Watkins, G. Brown, and K. Blumenstock, "Control of integrated radial and axial magnetic bearings," in Proceedings of the 33rd Southeastern Symposium on System Theory, pp. 1-5, Athens, Ohio, USA, March 2001.

[20] K. A. Blumenstock and G. L. Brown, "Novel integrated radial and axial magnetic bearing," in Proceedings of the 7th International Symposium on Magnetic Bearings, pp. 467-472, Zürich, Switzerland, August 2000.

[21] P. Imoberdorf, C. Zwyssig, S. D. Round, and J. W. Kolar, "Combined radial-axial magnetic bearing for a $1 \mathrm{~kW}, 500,000 \mathrm{rpm}$ permanent magnet machine," in Proceedings of the 22nd Annual IEEE Applied Power Electronics Conference and Exposition (APEC '07), pp. 1434-1440, Anaheim, Calif, USA, March 2007.

[22] P. Imoberdorf, T. Nussbaumer, and J. W. Kolar, "Analysis of a combined radial-axial magnetic bearing for a high-speed drive system," in Proceedings of the 5th IET International Conference on Power Electronics, Machines and Drives (PEMD '10), pp. 1-6, Brighton, UK, April 2010.

[23] Z. Xie, H. Zhu, and Y. Sun, "Structure and control of AC-DC three-degree-of-freedom hybrid magnetic bearing," in Proceeding of 11th International Conference on Electrical Machines and Systems (ICEMS '05), vol. 3, pp. 1801-1806, Wuhan, China, 2008.

[24] P. T. McMullen, C. S. Huynh, and R. J. Hayes, "Combination radial-axial magnetic bearing," in Proceedings of the 7 th International Symposium on Magnetic Bearings, pp. 473-478, Zürich, Switzerland, August 2000.

[25] W. Ni, Q. Wu, D. Jin, X. He, and T. Zhang, "Study on measuring and control system of AC radial-axial hybrid magnetic bearing used in wind energy generation system," in Proceedings of the 31st Chinese Control Conference (CCC '12), pp. 6847-6850, Hefei, China, July 2012.

[26] U. J. Na, "Design and analysis of a new permanent magnet biased integrated radial-axial magnetic bearing," International Journal of Precision Engineering and Manufacturing, vol. 13, no. 1, pp. 133-136, 2012.

[27] L. Huang, G. Zhao, H. Nian, and Y. He, "Modeling and design of permanent magnet biased radial-axial magnetic bearing by extended circuit theory," in Proceedings of the International Conference on Electrical Machines and Systems (ICEMS '07), pp. 1502-1507, Seoul, South Korea, October 2007.

[28] W. Zhang, Y. Ruan, X. Diao, and H. Zhu, "Control system design for AC-DC three-degree-of-freedom hybrid magnetic bearing," Applied Mechanics and Materials, vol. 150, pp. 144-147, 2012. 


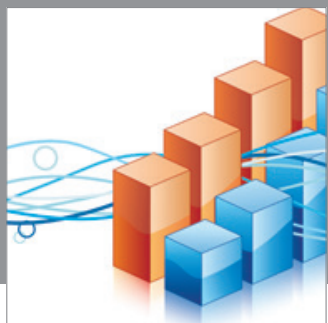

Advances in

Operations Research

mansans

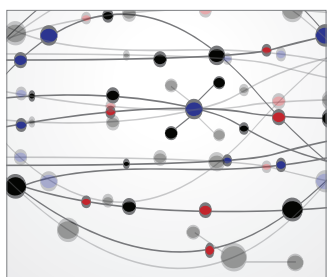

The Scientific World Journal
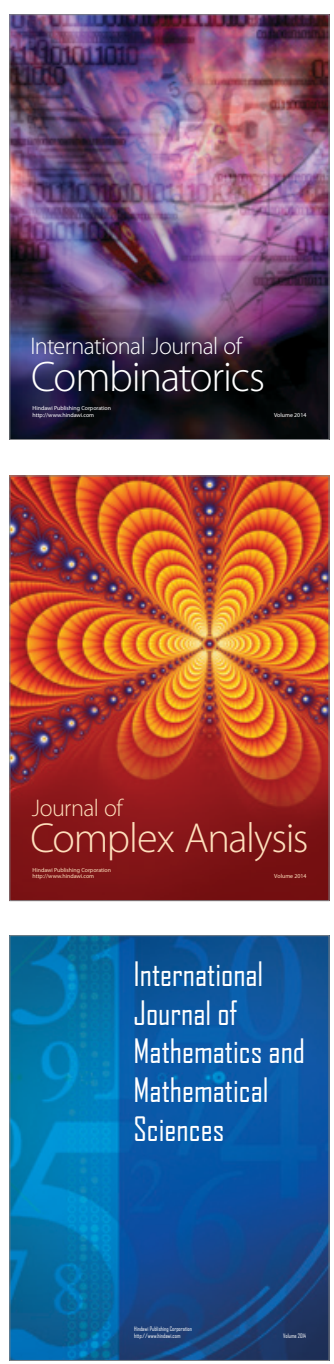
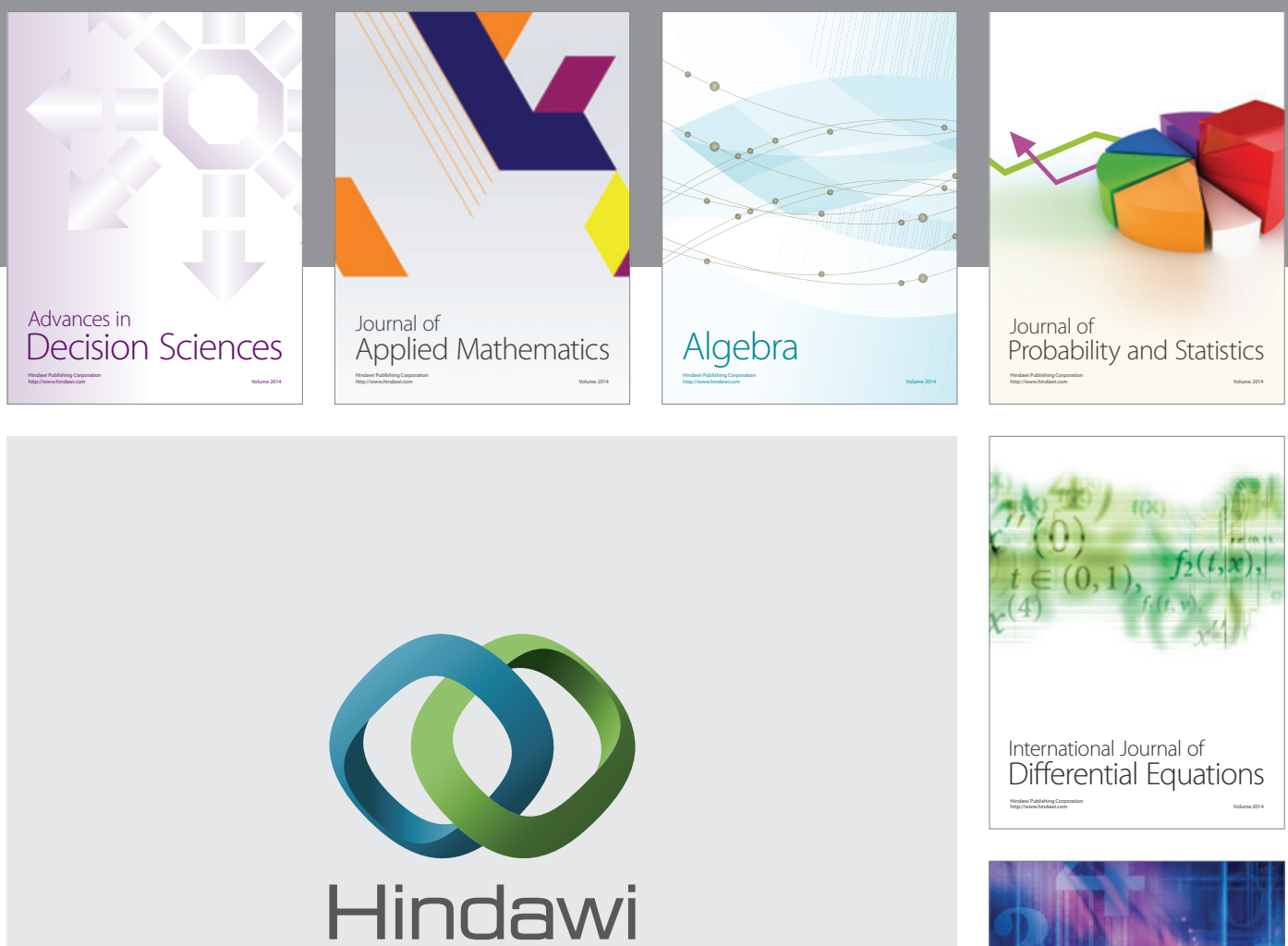

Submit your manuscripts at http://www.hindawi.com
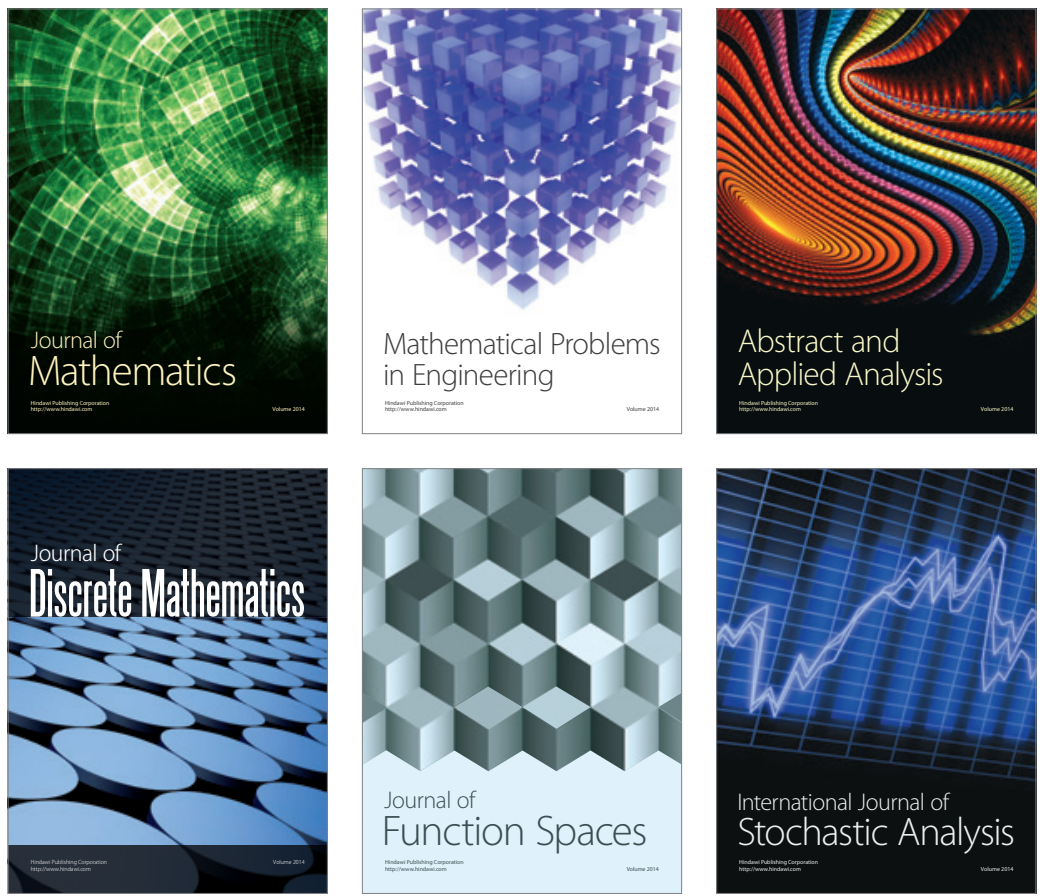

Journal of

Function Spaces

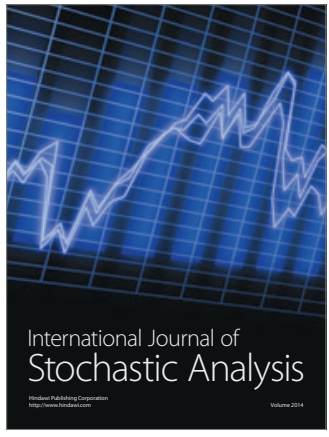

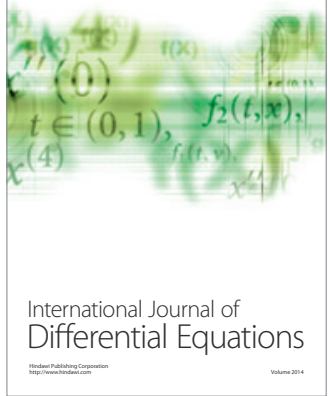
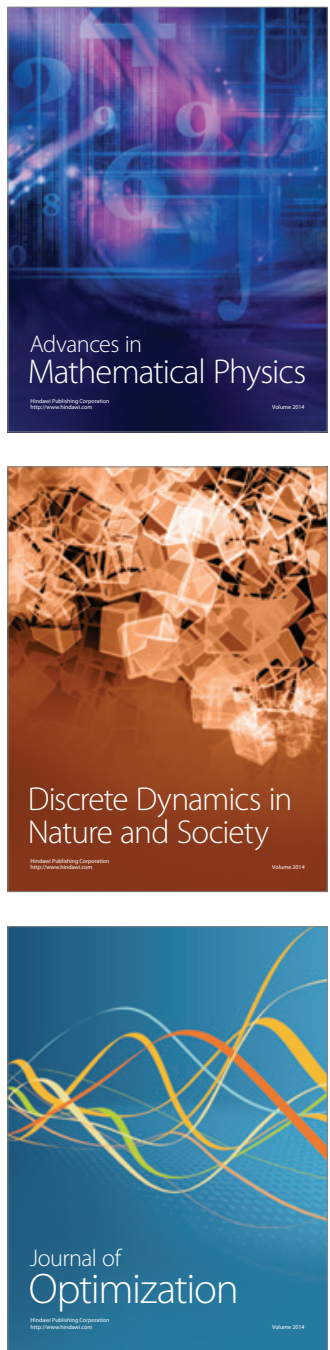